\title{
Hydrodynamic Limit of the Stationary Boltzmann Equation in a Slab
}

\author{
R. Esposito ${ }^{1}$, J.L. Lebowitz ${ }^{2}$, R. Marra ${ }^{3}$ \\ ${ }^{1}$ Dipartimento di Matematica, Università di Roma Tor Vergata, I-00133 Roma, Italy \\ 2 Mathematics and Physics Departments, Rutgers University, New Brunswick, NJ 08903, USA \\ 3 Dipartimento di Fisica, Università di Roma Tor Vergata, I-00133 Roma, Italy
}

Received: 20 March 1993

\begin{abstract}
We study the stationary solution of the Boltzmann equation in a slab with a constant external force parallel to the boundary and complete accommodation condition on the walls at a specified temperature. We prove that when the force is sufficiently small there exists a solution which converges, in the hydrodynamic limit, to a local Maxwellian with parameters given by the stationary solution of the corresponding compressible Navier-Stokes equations with no-slip boundary conditions. Corrections to this Maxwellian are obtained in powers of the Knudsen number with a controlled remainder.
\end{abstract}

\section{Introduction}

In this paper we continue our study of the derivation of hydrodynamic equations from the Boltzmann equation (BE), a problem which goes back to Hilbert [1]. The $\mathrm{BE}$ is believed to accurately describe the time evolution of rarefied gases on a "kinetic" scale intermediate between the microscopic and macroscopic [2]. To go from the BE to the macroscopic (hydrodynamic) descriptions the locally conserved density fields have to be slowly varying on the kinetic (to which we shall refer from now on as microscopic) scale but have sensible space variations over macroscopic distances. Let $\varepsilon$ be the ratio between microscopic and macroscopic space units (usually called the Knudsen number). It can be shown that the conserved densities, observed at microscopic times of order $\varepsilon^{-1}$, converge, as $\varepsilon \rightarrow 0$, to macroscopic fields whose time evolution is given by the solution of the Euler equations (EE) (at least when the latter have a smooth solution) $[3,4,5]$. This derivation of the EE in the above hydrodynamical (Euler) scaling limit is consistent with (indeed made possible by) the fact that the EE are themselves invariant under uniform space and time scaling.

Unfortunately there is no such scale invariance (and thus no such scaling limit) for the Navier-Stokes equations (NSE). The NSE are usually deduced, via the Chapman-Enskog expansion (see [6]), as corrections to the EE on the Euler time scale $\varepsilon^{-1}$ with viscosity coefficient and thermal conductivity of order $\varepsilon$. To describe situations which discriminate between the two equations when the Knudsen 
number $\varepsilon$ goes to zero we need to consider longer time scales, i.e. microscopic times of order $\varepsilon^{-2}$. On this time scale viscosity and thermal conductivity have finite effects, but the effect of the Euler term may now become very large making any rigorous mathematical analysis very difficult.

This problem can be controlled when the velocity field itself is of order $\varepsilon-$ in which case a kind of scale invariance is recovered for the NSE. It is then possible to get the incompressible NSE as a scaling limit from the BE (see [7-11]). To deal with the case of macroscopic velocities with non-vanishing Mach number it is necessary to consider situations in which some of the non-linear terms, which prevent invariance under scaling, are absent from the NSE due to the symmetry of the problem. Some partial results in this direction are given in [12].

Alternatively we can consider stationary situations in the presence of boundaries in which the time independent NSE have suitable scaling behavior. In this paper we consider such an example and show that the NS description is obtained as the scaling limit of the stationary solution of the BE. More precisely we consider a fluid in a channel with planar parallel boundaries subject to a constant force parallel to the boundary. Using the Euler equations the fluid velocity would keep on increasing linearly with time. For the NSE with no-slip boundary condition, on the other hand, the effect of the viscosity counteracts the force field and a stationary situation is reached, with the heat production balanced by a flow of energy to the thermal walls. Experiments, as well as molecular dynamics simulations [13], show that this situation can be reasonably described by the NSE.

The kinetic description of this problem in terms of a $\mathrm{BE}$ requires boundary conditions; a natural choice is to assume a complete accommodation coefficient. This means that any particle hitting the boundary is reflected with a velocity chosen at random according to a Maxwellian with variance corresponding to the fixed temperature of the walls. We confine ourselves to the fully symmetric solutions of the hydrodynamic and kinetic equations. The solution to the rescaled $\mathrm{BE}$ is then found like in [4] in terms of a truncated expansion in the Knudsen parameter $\varepsilon$ whose leading term is a Maxwellian with parameters given by the solution of the NSE.

The corrections to the leading order are given in the bulk by a modified truncated Hilbert expansion with a remainder. It is also necessary to introduce boundary layer terms to accommodate the expansion to the boundary conditions. This is accomplished along the lines of [14-17], but there are some extra difficulties arising from the force field.

Most of the paper is devoted to the proof of a bound on the remainder, which is obtained for a sufficiently small force field; this implies that the solution will match the expansion to any order, although, of course, no convergence of the expansion is provided or expected.

The outline of the paper is as follows. In Sect. 2 we present the problem and the basic results. Section 3 is devoted to the bulk and boundary layer expansions. The control of the boundary layer expansion requires the use of some well known results about the Milne problem (see [15-17]) and some estimates on the velocity derivatives of the boundary layer terms; these are given in Appendix B. There are many technical difficulties in estimating the remainder, which satisfies a weakly non-linear equation, due to the fact that for the stationary problem the only available a priori bound on the solution comes from the non-positivity of the quadratic form corresponding to the production of entropy in the linearized regime. Unfortunately the linearized Boltzmann operator has a non-trivial kernel 
so the control of the hydrodynamic component of the solution is very inefficient. This difficulty, combined with the high velocity difficulty already present in [4] and the low velocity difficulty typical of the stationary case make the estimates rather intricate. In Sect. 4 we construct the solution of the remainder equation as the limit of a sequence of solutions of approximating linear problems. The estimates for these solutions are obtained, like in [4], by decomposing them into a low and high velocity part, but the poor control of the hydrodynamic part at low velocities allows only $L_{2}$ bounds on the solution. The proof of these bounds is given in Sect. 5 . We then take advantage of the structure of the equation to improve the bounds to pointwise estimates on the solution which can be used to handle the non-linear terms. This is discussed in Sect. 6. Appendix A contains a short discussion of the solution of the macroscopic equations.

\section{Statement of Problem and Results}

a) Hydrodynamical Description. We consider the flow of a compressible viscous fluid in a three dimensional slab infinite (or periodic) in the $x$ and $z$ directions with planar boundaries perpendicular to the $y$ direction: top and bottom planes will correspond to $y=1$ and $y=-1$ respectively. The fluid is subject to a constant force parallel to the $x$-axis. A stationary state will be reached when the heat produced by the friction due to the relative motion of the gas under the action of the force is balanced by the energy loss to the walls maintained at a given temperature $T_{0}$.

Considering only situations which have the full symmetry of the problem the Navier-Stokes equations for this system, with a perfect gas equation of state, reduce to

$$
\begin{aligned}
\frac{d}{d y}(\rho T) & =0, \\
\frac{d}{d y}\left(\eta(T) \frac{d u}{d y}\right)+\rho F & =0, \\
\frac{d}{d y}\left(\kappa(T) \frac{d T}{d y}\right)+\eta(T)\left(\frac{d u}{d y}\right)^{2} & =0,
\end{aligned}
$$

where $u(y)$ is the $x$-component of the velocity field (the only non-vanishing one), $T(y)>0$ is the temperature field, $\rho(y)>0$ is the density of the fluid, $F>0$ is the constant intensity of the external field; $\eta(T)$ and $\kappa(T)$ are the viscosity and the thermal conductivity, which for gases depend only on $T$. The hydrostatic condition (2.1), stating the constancy of the pressure $\mathscr{P}$ given by the perfect gas law $\mathscr{P}=\rho T$, ensures that there is no flow in the $y$ direction.

We complete the system (2.1)-(2.3) with a no-slip boundary condition on the thermal walls at temperatures $T_{0}$, that is we assume

$$
u(-1)=u(1)=0 ; \quad T(-1)=T(1)=T_{0}>0 .
$$

Once the total mass per unit area $m=\int_{-1}^{1} \rho(y) d y$ is given, the problem is completely specified. The functions $\eta(T)$ and $\kappa(T)$ are assumed to be smooth and satisfy the conditions

$$
\eta(T) \geqq \eta_{0}>0 ; \quad \kappa(T) \geqq \kappa_{0}>0
$$

at least for $T \geqq T_{0}$. Under these conditions it is not difficult to prove the following 
Proposition 2.1. The system (2.1)-(2.5) has solutions in $C^{\infty}([-1,1])$, such that $T(y)>T_{0}$ for $y \in(-1,1)$. Moreover there is an $F_{0}>0$ such that, if $F \leqq F_{0}$ the solution is unique and for any positive $n$ there is a $c>0$ such that the following estimate holds:

$$
\sup _{y \in[-1,1]}\left[|u(y)|+\sum_{k=1}^{n}\left|u^{(k)}(y)\right|+\left|T(y)-T_{0}\right|+\sum_{k=1}^{n}\left|T^{(k)}(y)\right|\right]<c F,
$$

with $f^{(k)}$ denoting the $k^{\text {th }}$ derivative of $f$.

A sketch of the proof of Proposition 2.1 will be given in Appendix A.

b) Kinetic Description. To describe the situation in terms of the stationary BE we note first that Eqs. (2.1)-(2.3) are clearly invariant under a rescaling of spatial lengths by $\lambda$ and the force $F$ by $\lambda^{-2}$. We therefore introduce into the BE the Knudsen scale parameter $\varepsilon$ such that the width of the slab in microscopic units is $2 \varepsilon^{-1}$, and take the force field, in microscopic units, equal to $\varepsilon^{2} F$. Physically this means that the velocities of the particles of the gas are changed by a finite amount in times in which the effect of the viscosity are felt (i.e. times of order $\varepsilon^{-2}$ ).

This scaling of space by $\varepsilon$, from microscopic to macroscopic units, produces the following Boltzmann equation [7]:

$$
v_{y} \frac{\partial f}{\partial y}+\varepsilon F \frac{\partial f}{\partial v_{x}}=\frac{1}{\varepsilon} Q(f, f)
$$

where $y \in[-1,1], v=\left(v_{x}, v_{y}, v_{z}\right) \in \mathbb{R}^{3}$ denotes the velocity of the particles of the gas and $Q(f, f)(y, v)$ is the collision operator. We consider for concreteness the case of a hard sphere gas, for which $Q(f, g)$ is given for any $y$ by

$$
\begin{aligned}
Q(f, g)(v)= & \frac{1}{2} \int_{\mathbb{R}^{3} \times S_{+}^{2}} d v_{*} d w\left(v_{*}-v\right) \cdot \omega \\
& \times\left[f\left(v^{\prime}\right) g\left(v_{*}^{\prime}\right)+g\left(v^{\prime}\right) f\left(v_{*}^{\prime}\right)-f(v) g\left(v_{*}\right)-g(v) f\left(v_{*}\right)\right] .
\end{aligned}
$$

Here $S_{+}^{2}=\left\{\omega \in \mathbb{R}^{3}\right.$ s.t. $\left.\omega^{2}=1,\left(v_{*}-v\right) \cdot \omega \geqq 0\right\}, v^{\prime}$ and $v_{*}^{\prime}$ are the incoming velocities, $v$ and $v_{*}$ the outgoing velocities and $\omega$ the impact parameter of an elastic collision of hard spheres. In (2.8) the dependence on $y$ is implicit.

The thermal wall and the no-slip conditions are simulated by a Maxwell boundary condition with unit accommodation coefficient, temperature $T_{0}$ and vanishing mean velocity. Namely we assume, [6]

$$
\begin{gathered}
f(-1, v)=\alpha_{-} \bar{M}(v), \quad v_{y}>0, \\
f(1, v)=\alpha_{+} \bar{M}(v), \quad v_{y}<0,
\end{gathered}
$$

with

$$
\bar{M}(v)=\frac{1}{2 \pi T_{0}^{2}} \mathrm{e}^{-v^{2} / 2 T_{0}},
$$

normalized so that $\int_{v_{y} \lessgtr 0}\left|v_{y}\right| \bar{M}(v) d v=1$. In (2.9) the coefficients $\alpha_{ \pm}$are determined by the condition of impermeability of the walls, i.e.

$$
\left\langle v_{y} f\right\rangle=0 \text { for } y= \pm 1,
$$


and we use the notation

$$
\langle g\rangle \equiv \int_{\mathbb{R}^{3}} g(v) d v
$$

Hence $\alpha_{ \pm}$are given by

$$
\alpha_{ \pm}= \pm \int_{v_{y} \gtrless 0} v_{y} f( \pm 1, v) d v
$$

and can be interpreted as the flux of mass outgoing from the fluid toward the walls.

We now make explicit our restriction to flows which are symmetric under reflection of the $y$-axis. Denote by $\mathscr{R} v$ the reflected velocity, $\mathscr{R} v=\left(v_{x},-v_{y}, v_{z}\right)$, and by $\mathscr{R}^{*} f(y, v)=f(-y, \mathscr{R} v)$; we consider only solutions of (2.7) such that $\mathscr{R}^{*} f=f$. As a consequence of this reflection symmetry we have: $\int_{v_{y}>0} d v f(1, v) v_{y}$ $=-\int_{v_{y}<0} d v f(-1, v) v_{y}$, so that $\alpha_{+}=\alpha_{-}=\alpha$. Finally we require that the kinetic mass be equal to the hydrodynamic one, that is we assume the normalization condition

$$
\int_{-1}^{1} d y\langle f\rangle=m \text {. }
$$

The collision operator $Q(f, g)$ has the following properties:

i)

$$
\left\langle\hat{\chi}_{i} Q(f, g)\right\rangle=0 \text { for } i=0, \ldots, 4 \text {, }
$$

where

$$
\begin{aligned}
& \hat{\chi}_{0}(v)=1 ; \quad \hat{\chi}_{4}(v)=\frac{1}{2} v^{2}, \\
& \hat{\chi}_{1}(v)=v_{x}, \quad \hat{\chi}_{2}(v)=v_{y}, \quad \hat{\chi}_{3}(v)=v_{z} .
\end{aligned}
$$

ii)

$$
Q(f, f)=0 \quad \text { iff } \quad f(y, v)=M_{\rho, U, T}(v) \equiv \frac{\rho}{(2 \pi T)^{\frac{3}{2}}} \mathrm{e}^{-(v-U)^{2} / 2 T}
$$

with $\rho$ and $T$ positive and $U \in \mathbb{R}^{3}$. The function $M_{\rho, U, T}$ is called a local Maxwellian with parameters $\rho, T$ and $U$ depending on position.

Integrating (2.7) over velocities, it follows from (2.15) and (2.11) that any solution of (2.7) with the property (2.11) satisfies

$$
\left\langle v_{y} f\right\rangle=0 \text { for any } y \in[-1,1] .
$$

We denote by $M$ the Maxwellian $M_{\rho, U, T}$ with $T=T(y), \rho=\rho(y)$ and $U=(u(y), 0,0)$ given by the solution of $(2.1)-(2.4)$ and write, following $[4,7]$, the solution to (2.7)-(2.9) as

$$
f=M+\sum_{n=1}^{6} \varepsilon^{n} f_{n}+\varepsilon^{3} f_{R} .
$$

The functions $f_{n}$ 's will be specified in the next section as a combination of terms of a bulk expansion and of a boundary layer expansion. The remainder $f_{R}$, defined by (2.19), then has to solve the weakly non-linear equation

$$
v_{y} \frac{\partial f_{R}}{\partial y}+\varepsilon F \frac{\partial f_{R}}{\partial v_{x}}=\frac{1}{\varepsilon} \mathscr{L} f_{R}+\mathscr{L}^{1} f_{R}+\varepsilon^{2} Q\left(f_{R}, f_{R}\right)+\varepsilon^{3} A
$$


with

$$
\begin{gathered}
\mathscr{L} f \equiv 2 Q(M, f) \\
\mathscr{L}^{1} f_{R}=2 Q\left(\sum_{n=1}^{6} \varepsilon^{n-1} f_{n}, f_{R}\right)
\end{gathered}
$$

and $A$ a given function, specified in terms of the functions $f_{n}$ and their derivatives. Moreover, to satisfy the normalization condition (2.14), we must have

$$
\int d v d y f_{R}=0 .
$$

We summarize our results in the following

Theorem 2.2. Given $\rho, T$ and $u$ satisfying (2.1)-(2.4) and the force field $F$ small enough, then it is possible to determine uniquely functions $f_{n}, n=1, \ldots, 6$ and a remainder $f_{R}$ such that $f$, given by (2.19), is a solution to the $\mathrm{BE}(2.7),(2.9)$ and (2.14) for $\varepsilon$ sufficiently small. Moreover

$$
\begin{gathered}
\left\|f_{n}\right\|_{\infty}<c F, \\
\left\|f_{R}\right\|_{\infty}<c \varepsilon^{\frac{3}{2}} .
\end{gathered}
$$
(2.25).

In Sect. 3 we prove the estimate (2.24); Sects. $4-6$ are devoted to the proof of

Before going on to the proofs we make some remarks.

Remark 1 . In principle we could carry the sum in (2.19) up to any order $k \geqq 6$ and get a solution to the $\mathrm{BE}$ valid to $O\left(\varepsilon^{\ell}\right)$, for a suitable $\ell$ increasing with $k$.

Remark 2. Scaling the force field like $\varepsilon^{3}$ instead of $\varepsilon^{2}$, a factor $\varepsilon^{2}$ appears in front of $F$ in Eq. (2.7). The stationary macroscopic velocity field generated by such a force is of order $\varepsilon$. This corresponds, after a suitable rescaling, to the macroscopic flow being described by the incompressible NSE discussed in [7]. Equations (2.1)-(2.3) then reduce just to the linear equation (2.2), with constant $\rho$ and $T$, whose solution is the well known parabolic velocity profile. The arguments of this paper then provide the convergence of the stationary solution of the rescaled $\mathrm{BE}$ to the stationary solution of the incompressible NSE.

Remark 3. The planes $y=-1$ and $y=1$ could move along the $x$-direction with velocities $\mp V$ instead of being fixed. Our results remain true, as long as $|V|$ is small enough. For $F=0$ this corresponds to the well known planar Couette flow.

Remark 4. If the temperatures of the two planes are different the results of this paper do not apply because we then can no longer take advantage of reflection symmetry. Nevertheless, if the temperature difference is small, independently of $\varepsilon$, then we still have a unique solution of the hydrodynamic equations and the above results can be obtained by non-trivial modifications of our proof. This will be presented elsewhere.

\section{The Expansion}

Since the gradients of the hydrodynamical fields are non-vanishing on the boundaries, the behavior of the solution $f$ of the BE (2.7) is quite different in the bulk and in some thin (of order $\varepsilon$ on the macroscopic scale) region near the boundaries 
(kinetic boundary layer). To discriminate between these two behaviors we split the functions $f_{n}$ into the sum $B_{n}+b_{n}$ so that the expansion in $\varepsilon$ with coefficients $B_{n}$ represents a good approximation to $f$ away from the boundaries, while $b_{n}$ are boundary layer corrections.

The main properties of the $f_{n}$ 's are summarized in Proposition 3.1 below, in which the relevant norms are

$$
|f|_{r}=\sup _{y \in[-1,1]} \sup _{v \in \mathbb{R}^{3}}(1+|v|)^{r}|f(y, v)|
$$

for any non-negative integer $r$.

Proposition 3.1. Given $\rho, T$ and $u$ satisfying (2.1)-(2.4), and $F$ small enough, it is possible to determine constants $\alpha_{n}$ and functions $\gamma_{n, \varepsilon}^{ \pm}$of the velocity, exponentially small with $\varepsilon^{-1}$, uniformly in $v$, in such a way that the functions $f_{n}$ satisfy the following conditions:

i)

$$
f_{n}=B_{n}+b_{n}^{+}+b_{n}^{-}, \text {for } n=1, \ldots, 6
$$

ii)

with $B_{n}$ satisfying (3.11)-(3.13) and $b_{n}^{ \pm}$solutions of Eqs. (3.15)-(3.17) below.

$$
\left\langle v_{y} f_{n}\right\rangle=0 \text { for } y \in[-1,1] \text {. }
$$

iii)

$$
\int_{-1}^{1} d y\left\langle f_{n}\right\rangle=0 .
$$

iv)

$$
\begin{gathered}
f_{n}(-1, v)=\alpha_{n} M(-1, v)+\gamma_{n, \varepsilon}^{-}(v), \quad v_{y}>0, \\
f_{n}(1, v)=\alpha_{n} M(1, v)+\gamma_{n, \varepsilon}^{+}(v), \quad v_{y}<0 .
\end{gathered}
$$

v) There is for any positive $r, a$ constant $c$ such that:

$$
\begin{aligned}
& \left|M^{-\frac{1}{2}} f_{n}\right|_{r}<c F, \\
& \left|M^{-\frac{1}{2}} A\right|_{r}<c F,
\end{aligned}
$$

with $A$ defined in (3.19) below satisfying

vi)

$$
\langle A\rangle=0 \text { for } y \in[-1,1] \text {. }
$$

Remark. It follows by reflection symmetry that

$$
\begin{gathered}
f_{n}=\mathscr{R}^{*} f_{n}, \quad \gamma_{n, \varepsilon}^{-}(v)=\gamma_{n, \varepsilon}^{+}(\mathscr{R} v) \text { for } n=1, \ldots, 6, \\
\mathscr{R}^{*} A=A .
\end{gathered}
$$

The bulk terms $B_{n}$ satisfy the following set of conditions, corresponding to a modified Hilbert expansion:

$$
\begin{gathered}
v_{y} \frac{\partial M}{\partial y}=\mathscr{L} B_{1} \\
v_{y} \frac{\partial B_{1}}{\partial y}+F \frac{\partial M}{\partial v_{x}}=\mathscr{L} B_{2}+Q\left(B_{1}, B_{1}\right),
\end{gathered}
$$


and for $n=3, \ldots, 6$,

$$
v_{y} \frac{\partial B_{n-1}}{\partial y}+F \frac{\partial B_{n-2}}{\partial v_{x}}=\mathscr{L} B_{n}+\sum_{\substack{k, m \geqq 1 \\ k+m=n}} Q\left(B_{k}, B_{m}\right)
$$

with $\mathscr{L} f$ given by (2.21).

The boundary layer corrections $b_{n}$ will turn out to go to zero exponentially fast on distances of order $\varepsilon$ from the boundary. They are best described in terms of the rescaled variables $y^{\prime \prime}=\varepsilon^{-1}(1-y) ; y^{\prime}=\varepsilon^{-1}(y+1)$ with values in $\left[0,2 \varepsilon^{-1}\right]$, such that

$$
b_{n}=b_{n}^{+}\left(y^{\prime \prime}\right)+b_{n}^{-}\left(y^{\prime}\right) .
$$

We require the $b_{n}^{-}$to solve the following set of equations:

$$
\begin{gathered}
v_{y} \frac{\partial}{\partial y^{\prime}} b_{1}^{-}=\mathscr{L}_{0} b_{1}^{-}, \\
v_{y} \frac{\partial}{\partial y^{\prime}} b_{2}^{-}=\mathscr{L}_{0} b_{2}^{-}+2 Q\left(B_{1}, b_{1}^{-}\right)+Q\left(b_{1}^{-}, b_{1}^{-}\right)+2 Q\left(\Delta M, b_{1}^{-}\right)+Q\left(b_{1}^{-}, b_{1}^{+}\right),
\end{gathered}
$$

and for $n=3, \ldots, 6$,

$$
\begin{aligned}
v_{y} \frac{\partial}{\partial y^{\prime}} b_{n}^{-} & +F \frac{\partial}{\partial v_{x}} b_{n-2}^{-}=\mathscr{L}_{0} b_{n}^{-}+2 Q\left(\Delta M, b_{n-1}^{-}\right) \\
& +\sum_{\substack{i, j \geqq 1 \\
i+j=n}}\left[2 Q\left(B_{i}, b_{j}^{-}\right)+Q\left(b_{i}^{-}, b_{j}^{-}\right)+Q\left(b_{i}^{-}, b_{j}^{+}\right)\right],
\end{aligned}
$$

where $\mathscr{L}_{0} f=2 Q\left(M_{0}, f\right), M_{0}=M_{\rho( \pm 1), 0, T( \pm 1)}$ and $\Delta M=\varepsilon^{-1}\left(M-M_{0}\right)$. The functions $b_{n}^{+}$will satisfy an analogous set of equations relative to the boundary $y=1$.

Note that $\Delta M$ as a function of $y^{\prime}$ is bounded in compact intervals uniformly in $\varepsilon$. In fact, by Proposition 2.1,

$$
\left|\Delta M\left(y^{\prime}, v\right)\right|=\left|M_{0}(v)\left[\frac{\partial \log \rho}{\partial y}-\tilde{v}_{x} T^{-1} \frac{\partial u}{\partial y}-\frac{T^{-2}}{2}\left(\tilde{v}^{2}-3 T\right) \frac{\partial T}{\partial y}\right]\right|_{y=y^{\star}} y^{\prime} \mid \leqq c F y^{\prime}
$$

with a suitable $y^{\star} \in[-1, y]$ and $\tilde{v}$ the vector $\left(v_{x}-u, v_{y}, v_{z}\right)$. The last step is a consequence of (2.6).

With such a choice of $f_{n}$ the remainder $f_{R}$ will satisfy Eq. (2.20) with $A$ given by

$$
\begin{gathered}
A=-\left[v_{y} \frac{\partial B_{6}}{\partial y}+\varepsilon F \frac{\partial f_{6}}{\partial v_{x}}+F \frac{\partial f_{5}}{\partial v_{x}}\right]+\left[2 Q\left(\Delta M, b_{6}^{-}+b_{6}^{+}\right)\right] \\
+\sum_{\substack{k, m \geqq 1 \\
k+m \geqq 7}} \varepsilon^{k+m-7} Q\left(f_{k}, f_{m}\right) .
\end{gathered}
$$

The mass flux condition (2.18) and the normalization condition (2.14) for $f$ are assured by requiring that the $f_{n}$ satisfy condition (3.3) and (3.4). These conditions and the boundary conditions which we will discuss later couple the bulk and boundary layer expansions. 
Before proceeding to determine these intertwined expansions, we give some well known properties of the linear Boltzmann operator $\mathscr{L}$ proven by $\operatorname{Grad}[18,19,20]$. These are needed in the sequel and establish notation.

The Linearized Boltzmann Operator. It is convenient to denote by $\mathbb{H}$ the space $L_{2}\left(\mathbb{R}^{3}\right)$ and by $(f, g)$ the scalar product in $\mathbb{H}$. For any $y \in[-1,1]$ the operator

$$
L f \equiv M^{-\frac{1}{2}} \mathscr{L}\left(M^{\frac{1}{2}} f\right)
$$

is an unbounded operator on $\mathbb{H}$, which can be written as

$$
L f=-v f+K f
$$

with $v$ the multiplication operator by the function

$$
v(y, v)=\int_{\mathbb{R}^{3} \times S_{+}^{2}} d v_{*} d w\left(v_{*}-v\right) \cdot \omega M\left(y, v_{*}\right)
$$

and $K$ defined by (3.21). For hard spheres there are positive constants $v_{0}$ and $v_{1}$ such that:

$$
v_{0}(1+|v|) \leqq v(y, v) \leqq v_{1}(1+|v|)
$$

Moreover $K$ is a bounded operator in $\mathbb{H}$, so that $L$ is well defined on the domain $\mathscr{D}_{L}=\{f \in \mathbb{H}$ s.t. $v f \in \mathbb{H}\}$.

We need some notation. For any $y \in[-1,1]$ define

$$
\begin{gathered}
\chi_{0}(v)=\frac{1}{\sqrt{\rho}} \\
\chi_{1}(v)=\frac{v_{x}-u}{\sqrt{T \rho}} \quad \chi_{2}(v)=\frac{v_{y}}{\sqrt{T \rho}} \quad \chi_{3}(v)=\frac{v_{z}}{\sqrt{T \rho}} \\
\chi_{4}(v)=\frac{\left(v_{x}-u\right)^{2}+v_{y}^{2}+v_{z}^{2}-3 T}{2 T \sqrt{3 \rho}}
\end{gathered}
$$

and

$$
\psi_{i}=\chi_{i} \sqrt{M} \quad i=0, \ldots, 4
$$

The set $\left\{\psi_{i}, i=0, \ldots, 4\right\}$ is an orthonormal set in $\mathbb{H}$. Let $\mathscr{V}$ be the linear span of $\left\{\psi_{i}, i=0, \ldots, 4\right\}, P$ the orthogonal projector on $\mathscr{V}$ and $\bar{P}$ the projector on $\mathscr{W}$, the orthogonal complement of $\mathscr{V}$ in $\mathbb{H}$. We will refer to the projections of a function $f$ on $\mathscr{V}$ and $\mathscr{W}$ as the hydrodynamic and non-hydrodynamic part of $f$ respectively. We summarize here the properties of the operator $L$ that will be used in the rest of the paper.

i)

$$
\begin{gathered}
L f=0 \quad \text { iff } f \in \mathscr{V}, \\
\left(\psi_{i}, L f\right)=0, \quad i=0, \ldots, 4
\end{gathered}
$$

for any $f \in \mathscr{D}_{L}$. 
ii) For any $f, g \in D_{L}$

$$
\begin{gathered}
(f, L g)=(L f, g), \\
(f, L f) \leqq-c_{1} \int_{\mathbb{R}^{3}}(1+|v|)(\bar{P} f)^{2} \\
|(f, L g)| \leqq c_{2} \int_{\mathbb{R}^{3}}(1+|v|)|f g|
\end{gathered}
$$

for suitable positive constants $c_{1}$ and $c_{2}$.

iii) There is a positive constant $c$ such that

$$
\sup _{v \in \mathbb{R}^{3}}(1+|v|)^{r}|K f(v)| \leqq c \sup _{v \in \mathbb{R}^{3}}(1+|v|)^{r-1}|f(v)|
$$

for $r \geqq 1$,

$$
\begin{gathered}
\sup _{v \in \mathbb{R}^{3}}|K f(v)| \leqq c\left(\int_{\mathbb{R}^{3}}|f(v)|^{2} d v\right)^{\frac{1}{2}} \\
\int_{\mathbb{R}^{3}}|K f(v)|^{2} d v \leqq c \int_{\mathbb{R}^{3}}|f(v)|^{2} d v
\end{gathered}
$$

iv) The operator $K$ is compact on $\mathbb{H}$.

v) The equation

$$
L f=g
$$

is uniquely solvable for $g \in \mathscr{W}$. Denoting by $L^{-1}$ the inverse of the restriction of $L$ to $\mathscr{W}, L^{-1} g$ is in $\mathscr{W}$ and the general solution to (3.34) has the form

$$
\begin{gathered}
f=L^{-1} g+t, \quad t \in \mathscr{V}, \\
\sup _{v \in \mathbb{R}^{3}}(1+|v|)^{r}\left|L^{-1} g(v)\right| \leqq c \sup _{v \in \mathbb{R}^{3}}(1+|v|)^{r}|g(v)|, \\
\sup _{v \in \mathbb{R}^{3}}(1+|v|)^{r}\left|D_{v}^{\ell} L^{-1} g(v)\right| \leqq c \sup _{v \in \mathbb{R}^{3}}(1+|v|)^{r}\left|D_{v}^{\ell} g(v)\right|
\end{gathered}
$$

for any $r \geqq 0, \ell \geqq 1$ and a suitable constant $c, D_{v}^{\ell}$ denoting any partial derivative of order $\ell$ with respect to $v$. The estimate (3.37) is not contained in [20], but can be obtained by similar arguments (see [21]).

In the following we will also need the slightly different orthonormal set $\left\{\psi_{0}^{i}\right.$, $i=0, \ldots, 4$ in $\mathbb{H}\}$, defined by (3.24) and (3.25) with the following modifications: $\rho$, $T$ and $M$ are replaced by $\rho_{0}, T_{0}$ and $M_{0}$ respectively and $u=0$ because of the no-slip condition. We call $\mathscr{V}_{0}$ the linear span of $\psi_{0}$ and $P_{0}$ the associated orthogonal projectors. $\mathscr{W}_{0}$ is the orthogonal complement of $\mathscr{V}_{0}$ and $\bar{P}_{0}$ is the projector on $\mathscr{W}_{0}$. We will refer to the decomposition of an element of $\mathbb{H}$ on $\mathscr{V}_{0}$ and $\mathscr{W}_{0}$ as its hydrodynamical and non-hydrodynamical part without further specifications when it is clear from the context what we mean. We will also use the notation $L_{0} f=2 M_{0}^{-1 / 2} \mathscr{L}_{0}\left(M_{0}^{1 / 2} f\right)$. Of course $L_{0}$ has the same properties as $L$.

The Milne Problem. To deal with Eqs. (3.15)-(3.17) we have to consider the following linear transport half-space problem, which is a special case of the well 
known Milne problem:

$$
\begin{aligned}
v_{y} \frac{\partial}{\partial \tau} f & =L_{0} f+s, \quad 0 \leqq \tau<\infty, \\
f(0, v) & =\vartheta(v) \quad \text { for } v_{y}>0, \\
\left\langle v_{y} f(\tau, v)\right\rangle & =0 \text { for } \tau \in \mathbb{R}^{+}, \\
\lim _{\tau \rightarrow \infty} f & =f_{\infty} \in L_{\infty}\left(\mathbb{R}^{3}\right)
\end{aligned}
$$

for a given distribution $\vartheta$ of incoming particles at the boundary $\tau=0$ and a given source of particles $s$ such that

$$
\langle s\rangle=0 \text {. }
$$

This problem has been studied by several authors (see for example $[16,15,17]$ and references quoted therein).

\section{Theorem 3.2.} that

1) Suppose that for $r>3$ and some $\sigma^{\prime}>0$ there are finite constants $c_{1}$ and $c_{2}$ such

$$
\begin{gathered}
\sup _{v \in \mathbb{R}^{3}}(1+|v|)^{r}\left|M_{0}^{-\frac{1}{2}} \vartheta(v)\right|<c_{1}, \\
\sup _{\tau \in \mathbb{R}^{+}} e^{\sigma^{\prime} \tau} \sup _{v \in \mathbb{R}^{3}}(1+|v|)^{r}\left|M_{0}^{-\frac{1}{2}} S(\tau, v)\right|<c_{2} .
\end{gathered}
$$

Then there is a unique solution $f \in L_{\infty}\left(\mathbb{R}^{+} \times \mathbb{R}^{3}\right)$ to (3.38)-(3.41). Moreover there exist constants $c$ and $c^{\prime}$ such that $f$ verifies the conditions:

$$
\begin{gathered}
f_{\infty} \in \operatorname{Null} \mathscr{L}_{0}, \\
\sup _{\tau \in \mathbb{R}^{+}} e^{\sigma \tau} \sup _{v \in \mathbb{R}^{3}}(1+|v|)^{r} \mid M_{0}^{-\frac{1}{2}}\left(f(\tau, v)-f_{\infty}(v) \mid<c_{3}\right.
\end{gathered}
$$

for any $\sigma<c^{\prime}$.

2) Suppose that for $r>3, \ell \geqq 1$ and some $\sigma^{\prime}>0$,

$$
\sup _{v \in \mathbb{R}^{3}}(1+|v|)^{r}\left|M_{0}^{-\frac{1}{2}} \frac{\partial^{\ell} \vartheta}{\partial v_{x}^{\ell}}\right|+\sup _{\tau \in \mathbb{R}^{+}} e^{\sigma^{\prime} \tau} \sup _{v \in \mathbb{R}^{3}}(1+|v|)^{r}\left|M_{0}^{-\frac{1}{2}} \frac{\partial^{\ell} s}{\partial v_{x}^{\ell}}\right|<c_{\ell}
$$

for some constant $c_{\ell}$. There are finite constants $c$ and $c_{\ell}^{\prime}$ such that

$$
\sup _{\tau \in \mathbb{R}^{+}} e^{\sigma \tau} \sup _{v \in \mathbb{R}^{3}}(1+|v|)^{r}\left|M_{0}^{-\frac{1}{2}}\left[\frac{\partial^{\ell} f}{\partial v_{x}^{\ell}}-\frac{\partial^{\ell} f_{\infty}}{\partial v_{x}^{\ell}}\right]\right|<c_{\ell}^{\prime}
$$

for any $\sigma<c$.

For the proof of part 1) of Theorem 3.2 we refer to [16] where the case of hard spheres is discussed in detail. In [15] and [17] more general interactions are considered. The second part of Theorem 3.2 will be proven in Appendix B.

We are going to use (3.35) to solve (3.11)-(3.13) and Theorem 3.2 to solve (3.15)-(3.17). Actually Eqs. (3.15)-(3.17) are in the variables $y^{\prime}$ and $y^{\prime \prime}$ which take values in $\left[0,2 \varepsilon^{-1}\right]$, while in the Milne problem the variable $\tau$ is in $\mathbb{R}^{+}$. Of course it would be possible to reformulate the Milne problem in a bounded domain, but, in view of the fact that eventually $\varepsilon$ will go to 0 , it is more convenient to extend Eqs. (3.15)-(3.17) to $\mathbb{R}^{+}$by putting the functions $B_{n}$ and $\Delta M$ which will be computed in $\left[0,2 \varepsilon^{-1}\right]$, equal to 0 outside this interval. Since we do not exploit smoothness in 
$\tau$ of the solutions, the discontinuity introduced in this way is harmless. Of course for any finite $\varepsilon$ the only relevant part of the solution will be the restriction to $\left[0,2 \varepsilon^{-1}\right]$.

To find the functions $B_{n}$ solving (3.11)-(3.13) we have to check the solvability conditions which follow from (3.27). Since the function $M^{-\frac{1}{2}} Q(f, g)$ is in $\mathscr{W}$ by (2.15), it is enough to check that $M$ and $B_{n}$ 's verify the following conditions for $i=0, \ldots, 4$ :

$$
\begin{aligned}
\left\langle\hat{\chi}_{i} v_{y} \frac{\partial M}{\partial y}\right\rangle & =0, \\
\left\langle\hat{\chi}_{i}\left[v_{y} \frac{\partial B_{1}}{\partial y}+F \frac{\partial M}{\partial v_{x}}\right]\right\rangle & =0,
\end{aligned}
$$

and for $3 \leqq n \leqq 6$

$$
\left\langle\hat{\chi}_{i}\left[v_{y} \frac{\partial B_{n-1}}{\partial y}+F \frac{\partial B_{n-2}}{\partial v_{x}}\right]\right\rangle=0 .
$$

Condition (3.49) is satisfied because of (2.1). Hence we can solve (3.11) to find $B_{1}$. We have, by (3.35),

$$
B_{1}(y, v)=\mathscr{L}^{-1}\left[v_{y} \frac{\partial M}{\partial y}\right]+t_{1}(y, v)
$$

with the functions

$$
t_{1}(y, v)=\sum_{i=0}^{4} p_{i}^{(1)}(y) \psi_{i} M^{\frac{1}{2}}
$$

to be determined and $\mathscr{L}^{-1} f=M^{\frac{1}{2}} L^{-1}\left(M^{-\frac{1}{2}} f\right)$.

The explicit expression of $\mathscr{L}^{-1}\left[v_{y} \partial_{y} M\right]$ is (see for example [6])

$$
\bar{P}\left[\Theta_{1} \tilde{v}_{x} v_{y} \frac{\partial u}{\partial y}+\Theta_{2}\left(\tilde{v}^{2}-3 T\right) v_{y} \frac{\partial T}{\partial y}\right],
$$

where the $\Theta_{i}$ are some positive functions of $\tilde{v}^{2}$. Substituting now (3.52) and (3.54) in (3.50) the latter will be satisfied if (2.2), (2.3) and (2.5) hold with $\eta(T)$ and $\kappa(T)$ given in terms of $\Theta_{1}$ and $\Theta_{2}$. Moreover we have

$$
\frac{d}{d y}\left[p_{0}^{(1)}+\frac{5}{2 \sqrt{3}} p_{4}^{(1)}\right]=0, \quad \frac{d}{d y} p_{2}^{(1)}=0 .
$$

From (3.54) we see that, since $\partial_{y} u$ and $\partial_{y} T$ do not vanish on the boundary, $B_{1}$ does not vanish either and the terms $b_{1}^{ \pm}$have to be introduced to compensate $B_{1}$. To satisfy the boundary condition for $y=-1$ we have to choose $b_{1}^{-}$to be the solution to (3.15) with boundary condition in $y^{\prime}=0$ given by the value of $\mathscr{L}^{-1}\left[v_{y} \partial_{y} M\right]$ for $y=-1$. This corresponds to solving the problem (3.38)-(3.41) with $s=0$ and $\vartheta=\left.\mathscr{L}^{-1}\left[v_{y} \partial_{y} M\right]\right|_{y=-1}$. Let $\tilde{b}_{1}^{-}$be the solution of this problem. Then by (3.45) in Theorem 3.2 we see that $\tilde{b}_{1}^{-}$approaches, as $y^{\prime} \rightarrow \infty$, a function $q_{1}^{-}(v)$ in Null $\mathscr{L}$. Defining $b_{1}^{-}=\tilde{b}_{1}^{-}-q_{1}^{-}$, then $b_{1}^{-}$will be a solution to (3.15), with boundary condition

$$
\vartheta=\left.\mathscr{L}^{-1}\left[v_{y} \partial_{y} M\right]\right|_{y=-1}-q_{1}^{-},
$$

which goes to zero as $y^{\prime}$ goes to $\infty$. In the same way we construct $b_{1}^{+}$which is related to $b_{1}^{-}$in an obvious way by reflection symmetry. 
The above construction gives at $y=-1$ :

$$
f_{1}\left(-1, v_{y}>0\right)=t_{1}(-1, v)+b_{1}^{+}\left(2 \varepsilon^{-1}, v\right)-q_{1}^{-}(v),
$$

and at $y=1$,

$$
f_{1}\left(1, v_{y}<0\right)=t_{1}(1, v)+b_{1}^{-}\left(2 \varepsilon^{-1}, v\right)-q_{1}^{+}(v),
$$

where the function $t_{1}$ will be determined later.

Notice that by $(3.45) q_{1}^{ \pm}(v)$ and $t_{1}( \pm 1, v)$ are both in Null $\mathscr{L}_{0}$, so that we can use $t_{1}( \pm 1, v)$ to compensate $q_{1}^{ \pm}(v)$. The terms $b_{1}^{+}\left(2 \varepsilon^{-1}, v\right)$ are exponentially small in $\varepsilon^{-1}$ by (3.46) and in fact they are the terms denoted by $\gamma_{1, \varepsilon}^{ \pm}$in (3.5). By (3.40) $\left\langle v_{y} b_{1}^{ \pm}\right\rangle=0$ and, of course, $\left\langle v_{y} q_{1}^{ \pm}\right\rangle=0$ too. Therefore to satisfy (3.3) we have to choose

$$
p_{2}^{(1)}=0 \text {. }
$$

To determine completely the hydrodynamic part of $B_{1}$, that is the remaining coefficients $p_{i}^{(1)}, i \neq 2$, we use the linear differential equations obtained for them by means of the solvability conditions. First we solve (3.12) and obtain $B_{2}$ up to $t_{2}=\sum_{1=0}^{4} p_{i}^{(2)}(y) \psi_{i} M^{1 / 2}$. Then the solvability condition (3.51) with $n=3$ gives a system of three second order linear non-homogeneous differential equations for the unknown functions $p_{i}^{(1)}$ for $i=1,3$ and 4 . The coefficients $\Lambda_{i j}$ of the derivatives are given by $\Lambda_{i j}=\left(v_{y} \psi_{i}, L^{-1} v_{y} \psi_{j}\right)$. Since $L^{-1}$ is a strictly non-positive operator on $\mathscr{W},\left\|\Lambda_{i, j}\right\|$ are the coefficients of a $3 \times 3$ non-singular matrix. Hence the functions $p_{i}^{(1)}$ for $i=1,3$ and 4 are completely determined up to the values on the boundary. We write $q_{1}^{ \pm}=\sum_{i=0}^{4} q_{1, i}^{ \pm} \psi_{0}^{i} M_{0}^{\frac{1}{2}}$ and, for $i=1,3,4$, we choose the boundary values of $p_{i}^{(1)}$ as

$$
p_{i}^{(1)}( \pm 1)=q_{1, i}^{ \pm} .
$$

Then $p_{0}^{(1)}$ is determined by the first equation of (3.55) up to an additive constant that is chosen so that (3.4) is satisfied for $n=1$. Finally we obtain

$$
f_{1}\left( \pm 1, v_{y}>0\right)=\alpha_{1}^{ \pm} M_{0}+\gamma_{1, \varepsilon}^{ \pm}
$$

with $\alpha_{1}^{ \pm}=p_{0}^{(1)}( \pm 1)-q_{1,0}^{ \pm}$and $\gamma_{1, \varepsilon}^{ \pm}=b_{1}^{\mp}\left(2 \varepsilon^{-1}\right)$. In fact, by reflection symmetry $f_{1}=\mathscr{R} f_{1}$ and, in particular, $\alpha_{1}^{+}=\alpha_{1}^{-}$.

Now that $B_{1}$ and the non-hydrodynamical part of $B_{2}$ are completely determined, we can solve (3.16). To do that we have to use Theorem 3.2 with

$$
s=2 Q\left(B_{1}, b_{1}^{-}\right)+Q\left(b_{1}^{-}, b_{1}^{-}\right)+2 Q\left(\Delta M, b_{1}^{-}\right)+Q\left(b_{1}^{-}, b_{1}^{+}\right)
$$

and $\vartheta$ given, as before, by the difference between the value of the non-hydrodynamical part of $B_{2}$ in $y=-1$ and the limit value $q_{2}^{-}$of $\tilde{b}_{2}^{-}$. Reserving to Appendix B the check of the conditions of applicability of Theorem 3.2, we proceed as before to the construction of $b_{2}^{ \pm}$which vanish for $y^{\prime} \rightarrow \infty$. The determination of $t_{2}$ follows the same lines as the determination of $t_{1}$, and, in fact, we can repeat the above procedure step by step for the functions $f_{n}$.

The only difference in the $n^{\text {th }}$ step, for $n>2$, is due to the presence of the $v_{x}$-derivative of $b_{n-2}^{ \pm}$in the sources, arising from the presence of the external field. Their control is guaranteed by part 2 of Theorem 3.2.

We note that $t_{5}$ and $t_{6}$ are not completely determined by the above considerations. In fact (3.51) with $n=6$ only implies the conditions

$$
\frac{d}{d y}\left[p_{0}^{(5)}+\frac{5}{2 \sqrt{3}} p_{4}^{(5)}\right]=0, \quad \frac{d}{d y} p_{2}^{(5)}=0 .
$$


Since there is no compatibility condition to satisfy for $n>6$, there is also no differential equation to be satisfied by $t_{6}$. Nevertheless we still need to assume

$$
p_{2}^{(5)}=0, \quad p_{2}^{(6)}=0
$$

to satisfy (3.3) for $n=5,6$. Moreover we have to satisfy boundary conditions of the form

$$
\begin{aligned}
& p_{i}^{(5)}( \pm 1, v)=q_{5, i}^{ \pm}(v), \\
& p_{i}^{(6)}( \pm 1, v)=q_{6, i}^{ \pm}(v)
\end{aligned}
$$

for $i=1,3,4$. Therefore we choose $p_{i}^{(5)}$ and $p_{i}^{(6)}, i=1,3,4$, as constants matching the prescribed boundary values: they coincide by reflection symmetry. Moreover we use the arbitrary constant arising from the first equation in (3.63) to satisfy (3.4) with $n=5$. Finally we choose $p_{0}^{(6)}$ as a constant such that (3.4) is satisfied for $n=6$. The condition (3.8) is automatically satisfied by (3.19) since we have already chosen $p_{2}^{(6)}=0$. The estimate (3.6) easily follows by combining Proposition 2.1 with (3.36), (3.37), (3.46) and (3.48).

Finally we get the estimate (3.7) for $A$. The first three terms in (3.19) are controlled using (3.36), (3.37), (3.46) and (3.48).

To bound the last term of (3.19) we use (3.46) and the following estimate which has been proved by Grad [20] for any Maxwellian:

$$
\left|M^{-\frac{1}{2}} Q(R, S)\right|_{r-1} \leqq c\left|M^{-\frac{1}{2}} R\right|_{r}\left|M^{-\frac{1}{2}} S\right|_{r} .
$$

We use again (3.67) to estimate the remaining terms:

$$
\begin{aligned}
\left|M^{-\frac{1}{2}} 2 Q\left(\Delta M, b_{6}^{ \pm}\right)\right|_{r-1} & \leqq\left|M^{-\frac{1}{2}} \Delta M e^{-\sigma \tau}\right|_{r}\left|M^{-\frac{1}{2}} b_{6}^{ \pm} e^{\sigma \tau}\right|_{r} \\
& \leqq c F \sup _{\tau \in \mathbb{R}^{+}}\left|\tau e^{-\sigma \tau}\right| \leqq c F,
\end{aligned}
$$

where the second inequality is due to (3.46) and (3.18), since $M^{-\frac{1}{2}} \leqq c M_{0}^{-\frac{1}{2}}$.

This concludes the proof of Proposition 3.1.

\section{The Remainder}

In this section we construct the remainder $f_{R}$ and prove the crucial estimate (2.25). To fulfill the condition (2.23) it is convenient to put

$$
f_{R}=\alpha_{R} M+R
$$

so that (2.23) is satisfied if $\alpha_{R}$ is given by

$$
\alpha_{R}=-\frac{1}{m} \int d v d y R \text {. }
$$

In order to satisfy the boundary conditions (2.9) for $f$, taking into account the conditions (3.5) verified by the $f_{n}$ 's, we complete the non-linear problem (2.20) with the following boundary conditions:

$$
\begin{gathered}
f_{R}(-1, v)=\alpha_{R} M(-1, v)-\sum_{n=1}^{6} \varepsilon^{n-3} \gamma_{n, \varepsilon}^{-} \quad v_{y}>0, \\
f_{R}(1, v)=\alpha_{R} M(1, v)-\sum_{n=1}^{6} \varepsilon^{n-3} \gamma_{n, \varepsilon}^{+} \quad v_{y}<0 .
\end{gathered}
$$


The outgoing mass flux at the walls is thus determined in terms of the constants we have already fixed and $\alpha_{R}$ as

$$
\alpha=\rho_{0}\left(T_{0} / 2 \pi\right)^{1 / 2}\left(1+\sum_{n=1}^{6} \varepsilon^{n} \alpha_{n}+\varepsilon^{3} \alpha_{R}\right) .
$$

Using (2.20) we obtain the following equation for $R$, defined by (4.1),

$$
v_{y} \frac{\partial R}{\partial y}+\varepsilon F \frac{\partial R}{\partial v_{x}}=\frac{1}{\varepsilon} \mathscr{L} R+\mathscr{N} R+\varepsilon^{2} \tilde{Q}(R, R)+\varepsilon^{3} A,
$$

where the linear operator $\mathscr{N} R$ is the following modification of $\mathscr{L}^{1}$ :

$$
\mathscr{N} R=\mathscr{L}^{1} R-\frac{1}{m}\left[\mathscr{L}^{1} M-\varepsilon F \frac{\partial M}{\partial v_{x}}-v_{y} \frac{\partial M}{\partial y}\right] \int d v d y R .
$$

The non-linear term is given by

$$
\tilde{Q}(R, R)=Q(R, R)+\frac{2}{m} \mathscr{L} R \int d v d y R
$$

The boundary conditions for $R$ are

$$
\begin{aligned}
R(-1, v) & =-\sum_{n=1}^{6} \varepsilon^{n-3} \gamma_{n, \varepsilon}^{-} \quad v_{y}>0 \\
R(1, v) & =-\sum_{n=1}^{6} \varepsilon^{n-3} \gamma_{n, \varepsilon}^{+} \quad v_{y}<0 .
\end{aligned}
$$

The reflection symmetry of $R$ implies that $R(0, v)$ is an even function of $v_{y}$ and, as a consequence, $\left\langle v_{y} R(0, v)\right\rangle=0$. Therefore, integrating (4.5) on velocities, we see by (3.8) that $R$ satisfies the vanishing mass flux condition,

$$
\left\langle v_{y} R\right\rangle=0 \text {. }
$$

We prove the existence of the solution of a suitable integral form of Eqs. (4.5), (4.8) and (4.9) that will be specified in Sect. 6. It is constructed as a limit of the sequence $\left\{R_{k}, k \in \mathbb{N}\right\}$ of solutions of the approximate equations,

$$
v_{y} \frac{\partial R_{k}}{\partial y}+\varepsilon F \frac{\partial R_{k}}{\partial v_{x}}=\frac{1}{\varepsilon} \mathscr{L} R_{k}+\mathscr{N} R_{k}+\varepsilon^{2} \tilde{Q}\left(R_{k-1}, R_{k-1}\right)+\varepsilon^{3} A,
$$

with boundary conditions

$$
\begin{gathered}
R_{k}(-1, v)=-\sum_{n=1}^{6} \varepsilon^{n-3} \gamma_{n, \varepsilon}^{-} \quad v_{y}>0, \\
R_{k}(1, v)=-\sum_{n=1}^{6} \varepsilon^{n-3} \gamma_{n, \varepsilon}^{+} \quad v_{y}<0
\end{gathered}
$$

for $k \geqq 1$, with $R_{0}=0$.

To prove the convergence of the sequence $\left\{R_{k}, k \in \mathbb{N}\right\}$ we have to deal with a preliminary linear problem. We consider a function $D$ such that

$$
\mathscr{R}^{*} D=D, \quad\langle D\rangle=0
$$


and a function $\zeta_{-}$of velocity, defined for $v_{y}>0$. We look for the solution of the following linear boundary value problem:

$$
\begin{aligned}
v_{y} \frac{\partial R}{\partial y}+\varepsilon F \frac{\partial R}{\partial v_{x}} & =\frac{1}{\varepsilon} \mathscr{L} R+\mathscr{N} R+\varepsilon^{2} D, \\
R(-1, v) & =\zeta^{-} \quad v_{y}>0, \\
R(1, v) & =\zeta^{+} \quad v_{y}<0
\end{aligned}
$$

with $\zeta^{+}(v)=\zeta^{-}(\mathscr{R} v)$. The solution $R$ of (4.13), (4.14) will be estimated using the following norm:

$$
|f|_{r, \beta}=\sup _{y \in[-1,1]} \sup _{v \in \mathbb{R}^{3}}(1+|v|)^{r}|f(y, v)| \mathrm{e}^{\beta v^{2}},
$$

and the same notation will be used also for the norm of functions of velocity even if defined for incoming velocities only. We shall prove the following

Proposition 4.1. There are $\varepsilon_{0}>0, F_{1}>0$ and $\beta_{0}>0$ such that for any $\varepsilon<\varepsilon_{0}$, $F<F_{1}$ and $\beta<\beta_{0}$ the solution to the linear problem (4.13), (4.14) is bounded as follows:

$$
|R|_{r, \beta} \leqq c \varepsilon^{\frac{1}{2}}|D|_{r-1, \beta}+c \varepsilon^{-2}\left|\zeta^{-}\right|_{r, \beta}
$$

for any $r \geqq 3$ and for some constant $c$.

We note that the constant $\beta_{0}$ will turn out to be any positive number smaller than $\left(4 \sup _{y} T(y)\right)^{-1}$, while $F_{1}$ may be smaller than $F_{0}$ introduced in Proposition 2.1. Sections 5 and 6 are devoted to the proof of Proposition 4.1.

To bound the function $R_{k}$ we choose

$$
D=\varepsilon A+\tilde{Q}\left(R_{k-1}, R_{k-1}\right),
$$

while $\zeta^{ \pm}$are given by the r.h.s. of (4.11). Both terms in (4.7) can be bounded by $\left|R_{k-1}\right|_{r, \beta}^{2}$, using (3.67) with the Maxwellian $\exp \left\{-2 \beta v^{2}\right\}$. By Proposition 3.1 the functions $\gamma_{n}^{ \pm}$are exponentially small in $\varepsilon^{-1}$, so that $\left|\zeta^{ \pm}\right|_{r, \beta}$ are bounded by $c e^{-c^{\prime} \varepsilon^{-1}}$. Hence, for any $k>0$ and for any $r \geqq 3$ we have the estimate

$$
\left|R_{k}\right|_{r, \beta} \leqq c \varepsilon^{\frac{1}{2}}\left(\left|R_{k-1}\right|_{r, \beta}\right)^{2}+c \varepsilon^{\frac{3}{2}}|A|_{r, \beta}+c \varepsilon^{-2} e^{-c^{\prime} \varepsilon^{-1}} .
$$

This implies that, for $\varepsilon$ small enough, uniformly in $k$, for any $c^{\prime \prime}<c^{\prime}$,

$$
\left|R_{k}\right|_{r, \beta} \leqq c \varepsilon^{\frac{3}{2}}|A|_{r, \beta}+c e^{-c^{\prime \prime} \varepsilon^{-1}} .
$$

Denoting by $W_{k}=R_{k}-R_{k-1}$, for $k>1, W_{k}$ satisfies the equation

$$
\begin{aligned}
v_{y} \frac{\partial W_{k}}{\partial y}+\varepsilon F \frac{\partial W_{k}}{\partial v_{x}}=\frac{1}{\varepsilon} \mathscr{L} W_{k}+\mathscr{N} W_{k}+\varepsilon^{2} \tilde{Q}\left(R_{k-1}+R_{k-2}, W_{k-1}\right), \\
W_{k}(-1, v)=0 \quad v_{y}>0 \\
W_{k}(1, v)=0 \quad v_{y}<0 .
\end{aligned}
$$

In (4.20) we used the notation $\tilde{Q}(R, S)=Q(R, S)+\alpha_{S} \mathscr{L} R+\alpha_{R} \mathscr{L} S$. Hence, by choosing $D=\tilde{Q}\left(R_{k-1}+R_{k-2}, W_{k-1}\right)$ and $\zeta^{ \pm}=0$, using again Proposition 4.1, (3.67) and (4.19), it follows that

$$
\left|W_{k}\right|_{r, \beta} \leqq c \varepsilon^{2}\left|W_{k}\right|_{r-1, \beta} .
$$


Therefore for $\varepsilon \leqq \varepsilon_{0}$ the sequence $R_{k}$ converges and the limit satisfies (4.19). Let $R_{1}$ and $R_{2}$ be two solutions of (4.5), (4.8) with uniformly bounded $|\cdot|_{r, \beta}$. Then $W=R_{1}-R_{2}$ satisfies

$$
v_{y} \frac{\partial W}{\partial y}+\varepsilon F \frac{\partial W}{\partial v_{x}}=\frac{1}{\varepsilon} \mathscr{L} W+\mathscr{N} W+\varepsilon^{2} \tilde{Q}\left(R_{1}+R_{2}, W\right) .
$$

By Proposition 4.1 it follows that $|W|_{r, \beta} \leqq c \varepsilon^{\frac{1}{2}}|W|_{r, \beta}$, which implies uniqueness for $\varepsilon$ small enough. We have thus proved the following

Theorem 4.2. There are $\varepsilon_{0}>0, F_{1}>0$ and $\beta_{0}>0$ such that for any $\varepsilon<\varepsilon_{0}, F<F_{1}$ and $\beta<\beta_{0}$ the solution to the problem (4.5), (4.8), satisfies the bound

$$
|R|_{r, \beta} \leqq c \varepsilon^{\frac{3}{2}}|A|_{r, \beta}+c e^{-c^{\prime} \varepsilon^{-1}}
$$

for any $r \geqq 3$ and for some constant $c$.

Theorem 4.2 implies Theorem 2.2 and hence the solution of (2.7), (2.9) converges as $\varepsilon \rightarrow 0$ to the local Maxwellian with parameters solving the hydrodynamic equations (2.1)-(2.4).

\section{5. $L_{2}$-Estimates for the Linear Problem}

The natural way to deal with the linearized Boltzmann equation is to symmetrize $\mathscr{L}$ to get the operator $L$ in (3.20), and use the non-positivity (3.29) of $L$ to control the $\varepsilon^{-1} \mathscr{L}$ term in (4.5). On the other hand, when the Maxwellian is not space homogeneous, this procedure produces derivatives of the Maxwellian with respect to $y$ and $v_{x}$, which diverge as a polynomial in $v$ for large velocities. This difficulty has been solved by Caflisch in the time dependent case [4], by means of a decomposition of $R$ into a low velocity and high velocity part. The Caflisch method has to be modified here, since for the stationary problem the control of the hydrodynamic part of $R$ is worse. Like in [4], we introduce a global Maxwellian

$$
M_{*}=\left(2 \pi T_{*}\right)^{-3 / 2} \exp \left[-v^{2} / 2 T_{*}\right],
$$

where $T_{*}>\sup _{y \in[-1,1]} T(y)$ which is finite by Proposition 2.1. In this way $M_{*}$ $\geqq c M$ for all $(y, v)$ and some positive $c$.

We look for a solution of Eq. (4.13) in the form

$$
R=\sqrt{M} g+\sqrt{M_{*}} h,
$$

where the low velocity part $g$ and the high velocity part $h$ are defined as the solutions of the following system of coupled equations:

$$
\begin{gathered}
v_{y} \frac{\partial g}{\partial y}+\varepsilon F \frac{\partial g}{\partial v_{x}}+\left(\mu+\varepsilon F \mu^{\prime}\right) \hat{g}=\varepsilon^{-1} L g+\varepsilon^{-1} \chi_{\gamma} \sigma^{-1} K_{*} h+L^{1} \hat{g} \\
v_{y} \frac{\partial h}{\partial y}+\varepsilon F \frac{\partial h}{\partial v_{x}}+\varepsilon F \mu_{*}^{\prime} h+\left(\mu+\varepsilon F \mu^{\prime}\right) \sigma\left(\bar{g}+g_{2}\right) \\
=\varepsilon^{-1}\left(-v+\bar{\chi}_{\gamma} K_{*}\right) h+L_{*}^{1}\left[\sigma\left(\bar{g}+g_{2}\right)+h\right]+\varepsilon^{2} d
\end{gathered}
$$


The notation is:

$$
\begin{gathered}
\mu=v_{y} \frac{1}{2} \partial_{y} \log M, \quad \mu^{\prime}=\frac{1}{2} \partial_{v_{x}} \log M, \quad \mu_{*}^{\prime}=\frac{1}{2} \partial_{v_{x}} \log M_{*}, \quad \sigma=\sqrt{\frac{M}{M_{*}}}, \\
\chi_{\gamma}(v)= \begin{cases}1, & |v| \leqq \gamma \\
0, & |v|>\gamma\end{cases}
\end{gathered}
$$

$\bar{\chi}_{\gamma}=1-\chi_{\gamma}$,

$$
L_{*} f=M_{*}^{-1 / 2} 2 Q\left(M, M_{*}^{1 / 2} f\right)=\left(-v+K_{*}\right) f .
$$

$K_{*}$ is an integral operator analogous to $K$, for which the same properties (3.31)(3.33) hold (see [4]).

$$
\begin{gathered}
L^{1} f=M^{-1 / 2} \mathcal{N}\left(M^{1 / 2} f\right) \quad L_{*}^{1} f=M_{*}^{-1 / 2} \mathcal{N}\left(M_{*}^{1 / 2} f\right), \\
d=M_{*}^{-1 / 2} D .
\end{gathered}
$$

The low velocity part $g$ has been decomposed into a hydrodynamical part $\hat{g}+g_{2}$ and a non-hydrodynamic part $\bar{g}$,

$$
g=\hat{g}+g_{2}+\bar{g}, \quad \text { with } g_{2}=p_{2}(y) \psi_{2}, \quad \hat{g}=\sum_{j \neq 2} p_{j}(y) \psi_{j}
$$

We choose the following boundary conditions for $g$ and $h$ in Eqs. (5.3) and (5.4):

$$
\left\{\begin{array} { r l } 
{ g ( 1 , v ) = 0 } & { v _ { y } < 0 } \\
{ g ( - 1 , v ) = 0 } & { v _ { y } > 0 }
\end{array} \quad \left\{\begin{array}{rl}
h(1, v)=\zeta^{+} M_{*}^{-1 / 2} \equiv h_{+} & v_{y}<0 \\
h(-1, v)=\zeta^{-} M_{*}^{-1 / 2} \equiv h_{-} & v_{y}>0
\end{array} .\right.\right.
$$

Of course $h_{+}(v)=h_{-}(\mathscr{R} v)$. It is also convenient to consider $h_{ \pm}$extended to $\mathbb{R}^{3}$, putting it equal to 0 for $v_{y} \gtrless 0$.

The norm we are interested in is

$$
\|f\|=\left(\int_{[-1,1] \times \mathbb{R}^{3}} d y d v(1+|v|) f^{2}(y, v)\right)^{1 / 2} .
$$

We remark that in (5.4) the unbounded terms $\mu$ and $\mu^{\prime}$ are compensated by the factor $\sigma$ for large velocities while in (5.3) they appear as multipliers of $\hat{g}: \hat{g}$ has a good behavior for large velocities, but has a bad estimate in $\varepsilon$. This is the reason why we chose our decomposition in such a way that $\hat{g}$ does not appear in (5.4). Finally, the factor $\mu_{0}^{\prime}$ is also unbounded for large velocities but is a polynomial of degree 1 , so that it can be dominated in the norm (5.12). (For cross sections softer than hard spheres this would not be true because the natural norm to be used would include a power of $|v|$ less than 1.)

Theorem 5.1. There exist positive $\varepsilon_{0}, F_{1}$ and $c>0$ such that the solutions to Eqs. (5.3), (5.4) and (5.11) satisfy the bounds

$$
\begin{gathered}
\|\bar{g}\| \leqq \varepsilon^{2} c\left\|(1+|v|)^{-1} d\right\|+c \varepsilon^{-1 / 2}\left\|h_{-}\right\|, \\
\|\hat{g}\| \leqq \varepsilon c\left\|(1+|v|)^{-1} d\right\|+c \varepsilon^{-3 / 2}\left\|h_{-}\right\|, \\
\left\|g_{2}\right\|+\|h\| \leqq \varepsilon^{3} c\left\|(1+|v|)^{-1} d\right\|+c \varepsilon^{1 / 2}\left\|h_{-}\right\| .
\end{gathered}
$$

The proof is organized as follows: First we will obtain a bound for $\|g\|$ in terms of $\|h\|$. Using such a bound it will be possible to estimate $\|h\|$ in terms of $\left\|d(1+|v|)^{-1}\right\|$ and $\left\|h_{-}\right\|$. 
To estimate the operators $L^{1}$ and $L_{*}^{1}$ we will use the following estimate on the collision operator $Q$ proven in [22]: for any Maxwellian $M$ and for any $y \in[-1,1]$,

$$
\int_{\mathbb{R}^{3}} d v \frac{|Q(\sqrt{M} f, \sqrt{M} g)|^{2}}{(1+|v|) M} \leqq \int_{\mathbb{R}^{3}} d v(1+|v|)|f|^{2} \int_{\mathbb{R}^{3}} d v(1+|v|)|g|^{2} .
$$

(A) Estimates on $g$. The condition (4.9) together with (5.2) and the fact that $\bar{g}+\hat{g}$ is orthogonal to $\psi_{2}$, implies that

$$
\int d v\left(\sqrt{M} g_{2}+\sqrt{M}_{*} h\right) v_{y}=0 .
$$

As a consequence

$$
\sqrt{\rho T} p_{2}=-\int d v \sqrt{M}_{*} h v_{y} .
$$

Therefore, by Proposition 2.1 and the Schwarz inequality, we conclude that

$$
\left\|g_{2}\right\| \leqq C\|h\| \text {. }
$$

We now give estimates on $\bar{g}$ and on $\hat{g}$ separately.

a) Bound on $\bar{g}$. Multiplying Eq. (5.3) by $g$ and integrating it on $\mathbb{R}_{v}^{3}$ we have

$$
\frac{d}{d y}\left\langle v_{y} \frac{1}{2} g^{2}\right\rangle+\left\langle\left(\mu+\varepsilon F \mu^{\prime}\right) g \hat{g}\right\rangle=\varepsilon^{-1}(\bar{g}, L \bar{g})+\varepsilon^{-1}(\lambda, g)+\left(g, L^{1} \hat{g}\right),
$$

where $\lambda=\chi_{\gamma} \sigma^{-1} K_{*} h$. Let us observe that

$$
\left\langle\mu \hat{g}^{2}\right\rangle=\sum_{i, j \neq 2} p_{i} p_{j} \int d v \mu \psi_{i} \psi_{j}=0
$$

since, for $i, j \neq 2, \mu \psi_{i} \psi_{j}$, is an odd polynomial in $v_{y}$ times the Maxwellian, which is even in $v_{y}$. Moreover, using (3.11) in (4.6) we get

$$
\begin{aligned}
\mathscr{N} R= & \mathscr{L}^{1} R-\frac{1}{m}\left[2 Q\left(\sum_{n=2}^{6} \varepsilon^{n-1} f_{n}, M\right)\right. \\
& \left.+\mathscr{L}\left(b_{1}^{+}+b_{1}^{-}\right)-\varepsilon F \frac{\partial M}{\partial v_{x}}\right] \int d v d y R
\end{aligned}
$$

from which we see that the only term in $\mathcal{N} f$ that is not orthogonal to the collision invariants is $\varepsilon F \partial_{v_{x}} M$. Hence we have

$$
\int_{-1}^{1} d y\left|\left(\hat{g}, L^{1} \hat{g}\right)\right| \leqq c \varepsilon F\|\hat{g}\|^{2} .
$$

We remark that (5.21) and (5.23) are crucial to get an estimate on $g$.

Integrating Eq. (5.20) on $[-1,1]$ we get

$$
\mathscr{I}+\int d y d v\left(\mu+\varepsilon F \mu^{\prime}\right) g \hat{g}=\int d y d v\left[\varepsilon^{-1} \bar{g} L \bar{g}+\varepsilon^{-1} \lambda g+g L^{1} \hat{g}\right],
$$

where

$$
\mathscr{I}=\frac{1}{2}\left[\left\langle v_{y} g^{2}(1, v)\right\rangle-\left\langle v_{y} g^{2}(-1, v)\right\rangle\right] \geqq 0,
$$

using the boundary conditions (5.11) for $g$. 
Let us examine more closely the term involving $L^{1}$. By (5.16), (5.22) and (5.8), we have:

$$
\left\|(1+|v|)^{-1} L^{1} \hat{g}\right\| \leqq\left[\sum_{n=1}^{6} \varepsilon^{n-1}\left|M^{-1 / 2} f_{n}\right|_{3}+c \varepsilon F\right]\|\hat{g}\| .
$$

Hence, using (3.6), it follows that

$$
\int_{-1}^{1} d y\left|\left(\bar{g}, L^{1} \hat{g}\right)\right| \leqq c F\|\bar{g}\|\|\hat{g}\|
$$

To conclude the analysis of Eq. (5.24) consider the term $\left.\left\langle\left(\mu+\varepsilon F \mu^{\prime}\right) \hat{g} g\right)\right\rangle$. Taking into account that $\mu$ is a function of the derivatives of $\rho, T, u$ it follows by Proposition 2.1 and (5.10) that

$$
\|\mu \hat{g}\| \leqq c F\left(\int d y \sum_{j \neq 2} p_{j}^{2}\right)^{\frac{1}{2}} \leqq c F\|\hat{g}\| \sum_{j \neq 2}\left\|\psi_{j}\right\|^{2} \leqq c F\|\hat{g}\|,
$$

using the decay of $\hat{g}$ in $v$ to get the first inequality and the relation $p_{i}=\left(\hat{g}, \psi_{i}\right)$ to obtain the second inequality. In the same way it follows that $\left\|\mu^{\prime} \hat{g}\right\| \leqq c\|\hat{g}\|$. Hence, using (5.21) we have the following bound

$$
\int_{-1}^{1} d y\left|\left(\left(\mu+\varepsilon F \mu^{\prime}\right) \hat{g}, g\right)\right| \leqq c F\|\hat{g}\|\left(\|\bar{g}\|+\left\|g_{2}\right\|\right)+c \varepsilon F\|\hat{g}\|^{2} .
$$

Since $\lambda=\chi_{\gamma} \sigma^{-1} K_{*} h$ we have, by the $L_{2}$-boundedness of $K_{*}$ (see [4]),

$$
\|\lambda\| \leqq C_{\gamma}\|h\| \text {. }
$$

Finally, by (5.29), (5.30), (5.21), (5.23), (5.27) and (3.29) we have:

$$
\varepsilon \mathscr{I}+v_{0}\|\bar{g}\|^{2} \leqq C_{\gamma}\|h\|\|g\|+\varepsilon c F\|\hat{g}\|\|\bar{g}\|+\varepsilon^{2} F\|\hat{g}\|^{2} .
$$

b) Bound on $\hat{g}$. Multiplying Eq. (5.3) by $v_{y} \psi_{i}, i=0,1,3,4$ and integrating on $[-1, y] \times \mathbb{R}^{3}$, we have

$$
\begin{gathered}
\Phi_{i}(y)=\Phi_{i}(-1) \\
+\int_{-1}^{y} d y^{\prime} \int_{\mathbb{R}^{3}} d v v_{y} \psi_{i}\left[-\left(\mu+\varepsilon F \mu^{\prime}\right) \hat{g}-\varepsilon F \frac{\partial g}{\partial v_{x}}\right. \\
\left.+\varepsilon^{-1} L \bar{g}+\varepsilon^{-1} \lambda+L^{1} \hat{g}+v_{y}^{2} g \partial_{y} \psi_{i}\right],
\end{gathered}
$$

where $\Phi_{i}(y)=\left\langle v_{y}^{2} \psi_{i} g\right\rangle$.

First we give the estimate for $\Phi(-1)$. By the Schwarz inequality

$$
\left|\left\langle v_{y}^{2} \psi_{i} g(-1, v)\right\rangle\right| \leqq c\left\langle\left|v_{y}\right| g^{2}(-1, v)\right\rangle^{1 / 2}\left\langle\left|v_{y}\right|^{3} \psi_{i}^{2}\right\rangle^{1 / 2} \leqq c \mathscr{I}^{1 / 2},
$$

because $g(-1, v)=0$ for $v_{y}>0$. Therefore, taking into account the bound (5.31) for $\mathscr{I}$, we have

$$
\left|\Phi_{i}(-1)\right| \leqq c\left[\varepsilon^{-1} C_{\gamma}\|h\|\|g\|+F\|\bar{g}\|\|\hat{g}\|+\varepsilon F\|\hat{g}\|^{2}\right]^{\frac{1}{2}} .
$$

When the term involving the $v_{x}$-derivative in (5.32) is integrated by parts, then all the terms in Eq. (5.32) can be estimated using the Schwarz inequality and the bounds (5.26), (5.27), (5.29) and (3.30). The result is

$$
\begin{gathered}
\left|\Phi_{i}(y)\right| \leqq c\left[\varepsilon^{-1} C_{\gamma}\|h\|\|g\|+F\|\bar{g}\|\|\hat{g}\|+\varepsilon F\|\hat{g}\|^{2}\right]^{\frac{1}{2}} \\
\left.+c \varepsilon^{-1}\|\bar{g}\|+\varepsilon^{-1} C_{\gamma}\|h\|+c F\|g\|\right] .
\end{gathered}
$$


By the definition of $\Phi_{i}(y)$ we have:

$$
\Phi_{i}(y)=\left\langle v_{y}^{2} \psi_{i} g\right\rangle=\sum_{j \neq 2} A_{i j} p_{j}+\left\langle v_{y}^{2} \psi_{2}^{2}\right\rangle p_{2} \delta_{i, 2}+\left\langle v_{y}^{2} \psi_{i} \bar{g}\right\rangle,
$$

where $\delta_{i, j}$ is the Kronecker delta. The matrix $A$ whose elements are $A_{i j}=\left\langle v_{y}^{2} \psi_{i} \psi_{j}\right\rangle, i, j=0,1,3,4$ is non-singular. Hence the $p_{i}, i=0,1,3,4$ are determined by

$$
p_{i}=\sum_{j \neq 2} A_{i j}^{-1}\left[\Phi_{j}-\left\langle v_{y}^{2} \psi_{2}^{2}\right\rangle p_{2} \delta_{j, 2}-\left\langle v_{y}^{2} \psi_{j} \bar{g}\right\rangle\right]
$$

Therefore

$$
\|\hat{g}\|^{2} \leqq c \sum_{i \neq 2} \int_{-1}^{1} d y\left|p_{i}(y)\right|^{2} \leqq c \int_{-1}^{1} d y\left[\sum_{i \neq 2}\left[\Phi_{i}^{2}+C p_{2}^{2}\right]+c\|\bar{g}\|^{2} .\right.
$$

Hence, using the bound (5.35) for the $\Phi_{j}$ 's and (5.19) for $\left\|g_{2}\right\|$, we obtain

$$
\begin{aligned}
\|\hat{g}\| \leqq & c\left[\varepsilon^{-1} C_{\gamma}\|h\|\|g\|+F\|\bar{g}\|\|\hat{g}\|+\varepsilon F\|\hat{g}\|^{2}\right]^{\frac{1}{2}} \\
& +c \varepsilon^{-1}\|\bar{g}\|+\varepsilon^{-1} C_{\gamma}\|h\|+c F\|g\| .
\end{aligned}
$$

We simplify the right-hand side of Eq. (5.39) using the inequality

$$
|a b| \leqq \kappa a^{2}+(4 \kappa)^{-1} b^{2}
$$

for any $\kappa>0$, to bound the product $\varepsilon^{-1}\|h\|\|g\|$ with $\kappa\|g\|^{2}+(4 \kappa)^{-1} \varepsilon^{-2}\|h\|^{2}$. So we get

$$
\|\hat{g}\| \leqq c \varepsilon^{-1}\|\bar{g}\|+\varepsilon^{-1} C_{\gamma}\left(1+\frac{1}{4 \kappa}\right)\|h\|+(2 F+\kappa)\|g\| .
$$

Finally, choosing $\kappa$ and $F$ sufficiently small, we get

$$
\|\hat{g}\| \leqq c \varepsilon^{-1}\|\bar{g}\|+\varepsilon^{-1} C_{\gamma}\|h\| .
$$

Using (5.19) in (5.31) we have

$$
\|\bar{g}\|^{2} \leqq C_{\gamma}\|h\|(\|\bar{g}\|+\|\hat{g}\|+\|h\|)+c \varepsilon F\|\bar{g}\|\|\hat{g}\|+c \varepsilon^{2} F\|\hat{g}\|^{2} .
$$

Substituting (5.42) in (5.43), using again (5.40) we get

$$
\|\bar{g}\|^{2} \leqq\left(\sigma C_{\gamma}+c F\right)\|\bar{g}\|^{2}+\frac{c C_{\gamma}}{4 \sigma \varepsilon^{2}}\|h\|^{2}
$$

Hence, choosing $\sigma$ and $F$ sufficiently small, we have

$$
\|\bar{g}\| \leqq \varepsilon^{-1} C_{\gamma}\|h\| \text {. }
$$

In conclusion we have the following estimates for the hydrodynamic and nonhydrodynamic parts of $g$ :

$$
\begin{gathered}
\left\|g_{2}\right\| \leqq C_{\gamma}\|h\|, \\
\|\bar{g}\| \leqq \varepsilon^{-1} C_{\gamma}\|h\|, \\
\|\hat{g}\| \leqq \varepsilon^{-2} C_{\gamma}\|h\| .
\end{gathered}
$$

Estimates (5.46) and (5.47) will be used below to estimate $\|h\|$. 
(B) Estimates on $h$. The way to get bounds on $h$ is analogous to the one followed for $g$ but simpler since we do not need to control separately the hydrodynamic and the kinetic part. In fact in (5.4) the operator $L$ has been replaced by $\left(-v+\bar{\chi}_{\gamma} K_{*}\right)$ which has a trivial null space.

Multiplying Eq. (5.4) by $h$ and integrating on $[-1,1] \times \mathbb{R}^{3}$ we have

$$
\begin{aligned}
\mathscr{T} & +\int d y\left\langle\varepsilon F \mu_{*}^{\prime} h^{2}\right\rangle+\int d y\left\langle\left(\mu+\varepsilon F \mu^{\prime}\right) h \sigma\left(\bar{g}+g_{2}\right)\right\rangle \\
& =\int d y d v h\left[\varepsilon^{-1}\left(-v+\bar{\chi}_{\gamma} K_{*}\right) h+L_{*}^{1}\left[\sigma\left(\bar{g}+g_{2}\right)+h\right]+\varepsilon^{2} d\right],
\end{aligned}
$$

where $\mathscr{T}=\left\langle v_{y} h^{2}(1, v)\right\rangle-\left\langle v_{y} h^{2}(-1, v)\right\rangle \geqq-\left\langle\left|v_{y}\right| h_{-}^{2}\right\rangle$ by the boundary conditions (5.11).

We observe that, by the $L_{2}$-boundedness of the operator $K_{*}$ it follows that

$$
\left|\int d y\left\langle\bar{\chi}_{\gamma} h K_{*} h\right\rangle\right| \leqq\|h\|\left(\int d y\left\langle\bar{\chi}_{\gamma}\left(K_{*} h\right)^{2}(1+|v|)^{-1}\right\rangle\right)^{1 / 2} \leqq c\|h\|^{2}(1+\gamma)^{-1 / 2} \text {. }
$$

Hence

$$
\begin{aligned}
\varepsilon^{-1} v_{0}\|h\|^{2} \leqq & c\left[\varepsilon F\|h\|^{2}+F\|h\|\left(\|\vec{g}\|+\left\|g_{2}\right\|\right)+F\|h\|^{2}+\right. \\
& \left.+\varepsilon^{2}\|h\|\left\|d(1+|v|)^{-1}\right\|+\varepsilon^{-1}(1+\gamma)^{-1 / 2}\|h\|^{2}+\left\langle\left|v_{y}\right| h_{-}^{2}\right\rangle\right] .
\end{aligned}
$$

In fact the first integral in the 1.h.s of (5.49) has been bounded by $\varepsilon F\|h\|^{2}$, the second using the estimate $\left|\left(\mu+\varepsilon F \mu^{\prime}\right) \sigma\right| \leqq c F$. Moreover we used (3.6), (5.16), (5.22), (5.8) to get the bound

$$
\left\|(1+|v|)^{-1} L_{*}^{1}\left(h+\sigma\left(\bar{g}+g_{2}\right)\right)\right\|<c F\left(\|\bar{g}\|+\left\|g_{2}\right\|+\|h\|\right) .
$$

Then, using (5.46) and (5.47), we have

$$
\|h\|^{2} \leqq\left(C_{\gamma} F+c(1+\gamma)^{-\frac{1}{2}}\right)\|h\|^{2}+\varepsilon^{3}\|h\|\left\|d(1+|v|)^{-1}\right\|+c \varepsilon\left\langle\left|v_{y}\right| h_{-}^{2}\right\rangle .
$$

We fix $\gamma$ large enough to make $c(1+\gamma)^{-\frac{1}{2}} \leqq \frac{1}{3}$ and then $F$ sufficiently small to make $C_{\gamma} F \leqq \frac{1}{3}$, so that we can conclude that

$$
\|h\| \leqq c \varepsilon^{3}\left\|d(1+|v|)^{-1}\right\|+\varepsilon^{1 / 2}\left\|h_{-}\right\| .
$$

The proof of Theorem 5.1 then follows by combining (5.46)-(5.48) and (5.54).

\section{Pointwise Estimates for the Linear Problem}

Pointwise estimates of the solution of the linear problem are necessary to deal with the non-linear term of the Boltzmann operator. To get them we have to use an integral form of the equations for $g$ and $h$. This will give estimates of $L_{\infty}$-norms in terms of the norms used in the previous section.

Let us consider the equation

$$
v_{y} \frac{\partial f}{\partial y}+\varepsilon F \frac{\partial f}{\partial v_{x}}+\varepsilon^{-1} v f=\varepsilon^{-1} G
$$

with the boundary conditions

$$
f(-1, v)=f_{-}, v_{y}>0 ; \quad f(1, v)=f_{+}, v_{y}<0 .
$$


We use the following notation:

$$
\begin{gathered}
\Phi_{y, y^{\prime}}=\int_{y^{\prime}}^{y} d z v\left(z, v_{x}+\frac{\varepsilon F}{v_{y}}(z-y), v_{y}, v_{z}\right), \\
U_{\varepsilon} G(y, v)=\frac{1}{\varepsilon v_{y}} \int_{-1}^{y} d y^{\prime} G\left(y^{\prime}, v_{x}+\frac{\varepsilon F}{v_{y}}\left(y^{\prime}-y\right), v_{y}, v_{z}\right) \exp \left[-\frac{\Phi_{y, y^{\prime}}}{\varepsilon v_{y}}\right],
\end{gathered}
$$

for $v_{y}>0$ and

$$
U_{\varepsilon} G(y, v)=-\frac{1}{\varepsilon v_{y}} \int_{y}^{1} d y^{\prime} G\left(y^{\prime}, v_{x}+\frac{\varepsilon F}{v_{y}}\left(y^{\prime}-y\right), v_{y}, v_{z}\right) \exp \left[\frac{\Phi_{y^{\prime}, y}}{\varepsilon v_{y}}\right],
$$

for $v_{y}<0$.

$$
\begin{aligned}
& V_{\varepsilon}^{-} f^{-}=\chi\left(v_{y}>0\right) f^{-} \exp \left[-\frac{\Phi_{y,-1}}{\varepsilon v_{y}}\right], \\
& V_{\varepsilon}^{+} f^{+}=\chi\left(v_{y}<0\right) f^{+} \exp \left[\frac{\Phi_{1, y}}{\varepsilon v_{y}}\right] .
\end{aligned}
$$

The solution of Eq. (6.1), (6.2) can be written as

$$
f=V_{\varepsilon}^{+} f^{+}+V_{\varepsilon}^{-} f^{-}+U_{\varepsilon} G .
$$

Proposition 6.1. For any integer $r \geqq 0$ there is a constant $c$ such that the integral operator $U_{\varepsilon}$ satisfies the following inequality, uniformly in $\varepsilon$ :

$$
\left|U_{\varepsilon} G\right|_{r} \leqq c\left|\frac{G}{v}\right|_{r} .
$$

Proof. It is sufficient to prove (6.9) for $r=0$. In fact, let $G_{r}=G(1+|v|)^{r}$ and $f_{r}=U_{\varepsilon} G(1+|v|)^{r}$. Then the $f_{r}$ satisfy equations of the same type as Eq. (6.1),

$$
v_{y} \frac{\partial f_{r}}{\partial y}+\varepsilon F \frac{\partial f_{r}}{\partial v_{x}}+\varepsilon^{-1}\left(v-\varepsilon d_{r}\right) f_{r}=\varepsilon^{-1} G_{r},
$$

where $d_{r}=\varepsilon r F v_{x}[|v|(1+|v|)]^{-1},\left|d_{r}\right| \leqq \varepsilon F r$, with vanishing boundary conditions. Since for $\varepsilon$ small enough $v-\varepsilon d_{r} \geqq c v$ the proof for $r=0$ can be applied. Now for $r=0$ we have $\left(v_{y}>0\right)$

$$
\begin{aligned}
\left|\left(U_{\varepsilon} G\right)(y)\right| & \leqq \frac{1}{\varepsilon v_{y}} \int_{-1}^{y} d y^{\prime}\left|G\left(y^{\prime}, v_{x}+\frac{\varepsilon F}{v_{y}}\left(y^{\prime}-y\right), v_{y}, v_{z}\right)\right| \exp \left[-\frac{1}{\varepsilon v_{y}} \Phi_{y, y^{\prime}}\right] \\
& \leqq\left|\frac{G}{v}\right|_{0}^{y} d y^{\prime} \frac{1}{\varepsilon v_{y}} v\left(y^{\prime}, v_{x}+\frac{\varepsilon F}{v_{y}}\left(y^{\prime}-y\right), v_{y}, v_{z}\right) \exp \left[-\frac{1}{\varepsilon v_{y}} \Phi_{y, y^{\prime}}\right] \\
& \leqq\left|\frac{G}{v}\right|_{0}
\end{aligned}
$$

because $\int_{-1}^{y} d y^{\prime}\left(\varepsilon v_{y}\right)^{-1} v \exp \left\{-\left(\varepsilon v_{y}\right)^{-1} \Phi_{y, y^{\prime}}\right\}<1$.

The estimate for the case $v_{y}<0$ is obtained in the same way.

In the next proposition we use the following norm:

$$
N(f)=\sup _{y \in[-1,1]}\left(\int_{\mathbb{R}^{3}} d v|f(y, v)|^{2}\right)^{1 / 2} .
$$


Proposition 6.2. For any $\delta>0$ and for any $r \geqq 2$ there is a constant $C_{\delta}$ such that

$$
N\left(U_{\varepsilon} G\right) \leqq \frac{1}{\sqrt{\varepsilon}} C_{\delta}\left\|v^{-1 / 2} G\right\|+\delta|G|_{r}
$$

Proof. We give explicitly the proof only for $v_{y}>0$. Let us consider

$$
\begin{aligned}
& \int_{v_{y}>0} d v\left|U_{\varepsilon} G\right|^{2}= \\
& \quad \int d v\left[\int_{0}^{1+y} d t \frac{1}{\varepsilon v_{y}} G\left(y-t, v_{x}-\frac{\varepsilon F}{v_{y}} t, v_{y}, v_{z}\right) \exp \left[-\frac{1}{\varepsilon v_{y}} \Phi_{y, y-t}\right]\right]^{2} .
\end{aligned}
$$

In analogy to [22] we estimate the r.h.s. of (6.14) by decomposing it in the three parts $I_{1}, I_{2}, I_{3}$ defined as

$$
\begin{aligned}
& I_{1}=\int_{v_{y} \geqq \ell} d v\left|U_{\varepsilon} G\right|^{2}, \\
& I_{2}=\int_{0<v_{y} \leqq \ell} d v\left[\int_{\sigma}^{1+y} \frac{1}{\varepsilon v_{y}} G\left(y-t, v_{x}-\frac{\varepsilon F}{v_{y}} t, v_{y}, v_{z}\right) \exp \left[-\frac{1}{\varepsilon v_{y}} \Phi_{y, y-t}\right]\right]^{2}, \\
& I_{3}=\int_{0<v_{y} \leqq \ell} d v\left[\int_{0}^{\sigma} \frac{1}{\varepsilon v_{y}} G\left(y-t, v_{x}-\frac{\varepsilon F}{v_{y}} t, v_{y}, v_{z}\right) \exp \left[-\frac{1}{\varepsilon v_{y}} \Phi_{y, y-t}\right]\right]^{2} .
\end{aligned}
$$

By the Schwarz inequality we get

$$
\begin{aligned}
I_{1} \leqq & \int_{v_{y} \geqq \ell} d v \int_{0}^{1+y} d t \frac{1}{\varepsilon v_{y}} \frac{G^{2}}{v}\left(\left(y-t, v_{x}-\frac{\varepsilon F}{v_{y}} t, v_{y}, v_{z}\right)\right. \\
& \times \int_{0}^{1+y} d t \frac{v}{\varepsilon v_{y}} \exp -\left\{\frac{2}{\varepsilon v_{y}} \Phi_{y, y-t}\right\} \leqq c(\varepsilon \ell)^{-1}\left\|v^{-1} G\right\|^{2} .
\end{aligned}
$$

The bound on $I_{2}$ is obtained as follows. We observe that by Eq. (3.23),

$$
\frac{1}{\varepsilon v_{y}} \exp -\left\{\frac{2}{\varepsilon v_{y}} \Phi_{y, y-\sigma}\right\} \leqq c \sigma^{-1} .
$$

Setting $t^{\prime}=t-\sigma$ in (6.16) we are left with a term like $I_{1}$ times the 1.h.s. of (6.19). Hence, using (6.19) we have

$$
I_{2} \leqq c \sigma^{-1}\left\|v^{-1} G\right\|^{2} .
$$

The third term $I_{3}$ can be handled by noting that for $0 \leqq \beta \leqq 1$,

$$
\begin{aligned}
\frac{1}{\varepsilon v_{y}} \exp -\left\{\frac{1}{\varepsilon v_{y}} \Phi_{y, y-t}\right\} & \leqq c\left(\varepsilon v_{y}\right)^{\beta-1} \Phi_{y, y-t}^{-\beta} \\
& \leqq c\left(\varepsilon v_{y}\right)^{\beta-1}\left(v_{0} t\right)^{-\beta}
\end{aligned}
$$


Hence, by the Schwarz inequality,

$$
\begin{aligned}
I_{3} \leqq & c \frac{1}{\varepsilon^{2-2 \beta}} \int_{0}^{\sigma} d t_{1} \frac{1}{\left(v_{0} t_{1}\right)^{\beta}} \int_{0}^{\sigma} d t_{2} \frac{1}{\left(v_{0} t_{2}\right)^{\beta}} \int_{0<v_{y} \leqq \ell} \frac{d v_{y}}{v_{y}^{2-2 \beta}} \\
& \times\left[\int d v_{x} d v_{z} G^{2}\left(y-t_{1}, v_{x}-\frac{\varepsilon F}{v_{y}} t_{1}, v_{y}, v_{z}\right)\right]^{1 / 2} \\
& \times\left[\int d v_{x} d v_{z} G^{2}\left(y-t_{1}, v_{x}-\frac{\varepsilon F}{v_{y}} t_{2}, v_{y}, v_{z}\right)\right]^{1 / 2} .
\end{aligned}
$$

Then

$$
I_{3} \leqq c|G|_{r}^{2}\left(\frac{\sigma}{\varepsilon}\right)^{2-2 \beta} \int_{v_{y} \leqq \ell} \frac{d v_{y}}{v_{y}^{2-2 \beta}} \int d v_{x} d v_{z} \frac{1}{\left(1+v_{x}^{2}+v_{z}^{2}\right)^{r}},
$$

where we have fixed $\beta<1$ to make finite the integral over $t$. If we choose also $\beta>1 / 2$ and $r>1$ all the integrals in Eq. (6.23) are finite. Finally, since $\sigma$ is arbitrary, we can take $\sigma=\varepsilon$ getting

$$
I_{3} \leqq C \delta^{2}|G|_{r}^{2}
$$

with $\delta^{2}=\ell^{2 \beta-1}$.

Combining (6.18), (6.20) and (6.24) we get the result.

The regularizing properties of the operator $U_{\varepsilon}$ suggest to consider the following integral form of Eq. (4.5):

$$
\begin{aligned}
R= & U_{\varepsilon}\left[\frac{1}{\varepsilon}(\mathscr{L} R-v R)+\mathcal{N} R+\varepsilon^{2} \tilde{Q}(R, R)+\varepsilon^{3} A\right] \\
& -V_{\varepsilon}^{-}\left[\sum_{n=1}^{6} \varepsilon^{n-3} \gamma_{n, \varepsilon}^{-}\right]-V_{\varepsilon}^{+}\left[\sum_{n=1}^{6} \varepsilon^{n-3} \gamma_{n, \varepsilon}^{+}\right] .
\end{aligned}
$$

In fact we define a solution of (4.5), (4.8) as any solution of (6.25).

We consider now the integral versions of (5.3) and (5.4) that allow to prove estimates for the norm $|\cdot|_{r}$ of $g$ and $h$.

Proposition 6.3. There exist positive constants $c$ and $H_{\gamma}$ such that for any $r \geqq 2$ the solution $g$ of Eq. (5.3) verifies

$$
|g|_{r} \leqq c \sqrt{\varepsilon}\left\|d(1+|v|)^{-1}\right\|+H_{\gamma}|h|_{r}+c \varepsilon^{-2}\left|h_{-}\right|_{r} .
$$

Proof. We write Eq. (5.3) as

$$
v_{y} \frac{\partial g}{\partial y}+\varepsilon F \frac{\partial g}{\partial v_{x}}+\varepsilon^{-1} v g=\varepsilon^{-1}(K g+S)
$$

with

$$
S=-\varepsilon\left(\mu+\varepsilon F \mu^{\prime}\right) \hat{g}+\chi_{\gamma} \sigma^{-1} K_{*} h+\varepsilon L^{1} \hat{g} .
$$

Most of the proof is devoted to getting an estimate for $N(g)$ in terms of $N(h)$ and the $L_{2}$ norms of $g$ and $h$, whose control is assured by the results of Sect. 4 . Then we conclude by relating $N(h)$ to the $L_{\infty}$ norm of $h$. 
By Proposition 6.2 the solution of Eq. (6.27) verifies, for any $r \geqq 2$, the inequality

$$
N(g) \leqq \frac{1}{\sqrt{\varepsilon}} C_{\delta}\left\|v^{-1} K g\right\|+\delta|K g|_{r}+\frac{1}{\sqrt{\varepsilon}} C_{\delta}\left\|v^{-1} S\right\|+\delta|S|_{r} .
$$

The first and third terms on the right-hand side are easily estimated using Theorem 5.1 and the inequalities

$$
\left\|v^{-1} K g\right\| \leqq c\|g\| ;\left\|v^{-1} S\right\| \leqq \varepsilon F c\|\hat{g}\|+C_{\gamma}\|h\| .
$$

The second term in (6.29), is controlled by using the estimates (3.31) and (3.32), which allow to relate the supremum on velocities of $K f$ with the $L_{2}(d v)$ norm of $f$. In fact

$$
|K g|_{r} \leqq c \sup _{y \in[-1,1]} \sup _{v \in \mathbb{R}^{3}}(1+|v|)^{r-1}|g(y, v)|=c|g|_{r-1}
$$

by (3.31) and

$$
|K g|_{0}^{2} \leqq c \sup _{y \in[-1,1]} \int d v g^{2}(y, v)=c N(g)^{2}
$$

by (3.32).

Furthermore, since $g=U_{\varepsilon} K g+U_{\varepsilon} S$, we get by Proposition (6.1),

$$
\begin{aligned}
|g|_{r} & \leqq|K g|_{r}+|S|_{r} \leqq|g|_{r-1}+|S|_{r} \leqq \ldots \\
& \leqq|K g|_{0}+\sum_{k=0}^{r}|S|_{k} \leqq N(g)+\sum_{k=0}^{r}|S|_{k},
\end{aligned}
$$

where we have used (6.32) to get the last inequality.

Thus to complete the proof we have to estimate $S$ in the norm $|\cdot|_{r}$. We have

$$
|S|_{r} \leqq \varepsilon\left|\left(\mu+\varepsilon F \mu^{\prime}\right) \hat{g}\right|_{r}+\left|\chi_{\gamma} \sigma^{-1} K_{*} h\right|_{r}+\varepsilon\left|L^{1} \hat{g}\right|_{r} .
$$

By the exponential decay of $\hat{g}$ in velocities and (6.32) we have

$$
\left|\left(\mu+\varepsilon F \mu^{\prime}\right) \hat{g}\right|_{r} \leqq c F|\hat{g}|_{0} \leqq c F N(\hat{g}) .
$$

By the analogue of (6.32) for $K_{*}$ (see [4]) we have

$$
\begin{aligned}
\left|\chi_{\gamma} \sigma^{-1} K_{*} h\right|_{r} \leqq & \sup _{y \in[-1,1]} \sup _{v \in \mathbb{R}^{3}}\left[(1+|v|)^{r} \chi_{\gamma} \sigma^{-1}\right] \\
& \times \sup _{y \in[-1,1]} \sup _{v \in \mathbb{R}^{3}}\left|K_{*} h\right| \leqq H_{\gamma} N(h) .
\end{aligned}
$$

The operator $L^{1}$ satisfies the estimate

$$
\left|v^{-1} L^{1} f\right|_{r} \leqq c|f|_{r}
$$

analogous to the one proved in [4]. Using it we can estimate the last term:

$$
\left|L^{1} \hat{g}\right|_{r} \leqq c|\hat{g}|_{r+1} \leqq c \sup _{y \in[-1,1]}\left|\sum_{i \neq 2} p_{i}(y)\right| \leqq c|\hat{g}|_{0} \leqq c N(\hat{g}) .
$$

Collecting together (6.34), (6.35) and (6.38) we have the following bound for $S$ :

$$
|S|_{r} \leqq c \varepsilon N(\hat{g})+H_{\gamma} N(h) .
$$


By (6.31), (6.33) and (6.34), we have

$$
|K g|_{r} \leqq c N(g)+H_{\gamma} N(h) .
$$

Now we can estimate $N(g)$ by combining (6.29), (6.30), (6.39) and (6.40):

$$
N(g) \leqq c\left[\frac{1}{\sqrt{\varepsilon}} C_{\delta}\|g\|+\delta N(g)+H_{\gamma} N(h)+\frac{1}{\sqrt{\varepsilon}} C_{\delta} H_{\gamma}\|h\|\right] .
$$

Finally, taking into account estimates (5.46)-(5.48) and (5.54), we get, for $\delta$ small enough,

$$
\left.N(g) \leqq c\left[\sqrt{\varepsilon}\left\|d(1+|v|)^{-1}\right\|+\varepsilon^{-2}\left|h_{-}\right|_{3}\right]+H_{\gamma} N(h)\right] .
$$

On the other hand Proposition 6.1 implies

$$
\begin{aligned}
|g|_{r} & \leqq c\left|v^{-1} K g\right|_{r}+\left|v^{-1} S\right|_{r} \leqq c\left[N(g)+H_{\gamma} N(h)\right] \\
& \left.\leqq c\left[\sqrt{\varepsilon}\left\|d(1+|v|)^{-1}\right\|+\varepsilon^{-2}\left|h_{-}\right|_{3}\right]+H_{\gamma} N(h)\right],
\end{aligned}
$$

where the second inequality comes from (6.39) and (6.40) and the third one from (6.42).

For $r \geqq 2$ we have

$$
[N(h)]^{2} \leqq \sup _{y \in[-1,1]} \sup _{v \in \mathbb{R}^{3}}\left[h^{2}(y, v)(1+|v|)^{2 r}\right] \int_{\mathbb{R}^{3}} \frac{d v}{(1+|v|)^{2 r}} \leqq c|h|_{r}^{2} .
$$

Putting together estimates (6.43) and (6.44) we get the result (6.26).

Finally, we prove

Proposition 6.4. For any $r \geqq 3$ the solution $h$ of Eq. (5.4) verifies

$$
|h|_{r} \leqq c\left[\varepsilon^{\frac{3}{2}}\left\|d(1+|v|)^{-1}\right\|+\varepsilon^{3}\left|v^{-1} d\right|_{r}+\varepsilon^{-1}\left|h_{-}\right|_{r}\right] .
$$

Proof. Let us write Eq. (5.4) in the form

$$
h=U_{\varepsilon}\left(\bar{\chi}_{\gamma} K_{*} h\right)+U_{\varepsilon} Z+V_{\varepsilon}^{+} h_{+}+V_{\varepsilon}^{-} h_{-},
$$

where

$$
Z=-\mu_{0}^{\prime} \varepsilon^{2} F h-\varepsilon\left(\mu+\varepsilon F \mu^{\prime}\right) \sigma\left(\bar{g}+g_{2}\right)+\varepsilon L_{*}^{1}\left(h+\sigma\left(\bar{g}+g_{2}\right)\right)+\varepsilon^{3} d .
$$

Since $\left|V_{\varepsilon}^{ \pm} h_{ \pm}\right|_{r} \leqq\left|h_{-}\right|_{r}$, we have by Proposition 6.1,

$$
\begin{aligned}
|h|_{r} & \leqq c\left[\left|U_{\varepsilon}\left(v^{-1} \bar{\chi}_{\gamma} K_{*} h\right)\right|_{r}+\left|v^{-1} Z\right|_{r}+\left|h_{-}\right|_{r}\right] \\
& \leqq c\left[\frac{1}{1+\gamma}\left|K_{*} h\right|_{r}+\varepsilon^{2} F|h|_{r}+\varepsilon F\left(|\bar{g}|_{r}+\left|g_{2}\right|_{r}\right)+\varepsilon F|h|_{r}+\varepsilon^{3}\left|v^{-1} d\right|_{r}\right. \\
& \left.+\left|h_{-}\right|_{r}\right] \leqq c\left[\frac{1}{(1+\gamma)}|h|_{r}+\varepsilon|g|_{r}+\varepsilon^{3}\left|v^{-1} d\right|_{r}+\left|h_{-}\right|_{r}\right] .
\end{aligned}
$$

Choosing $\gamma$ in such a way that $\frac{1}{(1+\gamma)}<\frac{1}{3}$ we have, for $\varepsilon$ small enough,

$$
|h|_{r} \leqq c\left[\varepsilon|g|_{r}+\varepsilon^{3}\left|v^{-1} d\right|_{r}+\left|h_{-}\right|_{r}\right] \text {. }
$$


Finally, substituting the estimate for $g$ given by Proposition 6.3 in (6.49) and taking $\varepsilon$ small enough, we complete the proof of Proposition 6.4.

By using (6.49) to estimate $|h|_{r}$ in (6.26), the estimate $\left\|d(1+|v|)^{-1}\right\| \leqq c|d / v|_{3}$ and taking $\varepsilon$ small enough we then get the proof of Proposition 4.1.

\section{Appendix A}

To prove Proposition 2.1 we first consider Eqs. (2.1)-(2.4) with fixed pressure $\mathscr{P}$ instead of fixed total mass, by eliminating the density $\rho$ from (2.2) using (2.1). We then introduce the following sequence of approximate solutions:

$$
\begin{aligned}
\frac{d}{d y}\left(\eta\left(T_{n-1}\right) \frac{d u_{n}}{d y}\right)+\mathscr{P} F T_{n-1}^{-1} & =0, \\
\frac{d}{d y}\left(\kappa\left(T_{n-1}\right) \frac{d T_{n}}{d y}\right)+\eta\left(T_{n-1}\right)\left(\frac{d u_{n}}{d y}\right)^{2} & =0
\end{aligned}
$$

for $n \geqq 1, T_{0}$ being the value on the boundary and $u_{0}=0$. The boundary conditions are given by (2.4). By using induction, the maximum principle implies that

$$
T_{n}(y)>T_{0}
$$

for $y \in(-1,1)$. Hence

$$
\eta\left(T_{n}\right)>\eta_{0} \quad \text { and } \quad \kappa\left(T_{n}\right)>\kappa_{0}
$$

for $y \in(-1,1)$.

Multiplying (A.1) by $u_{n}$ and (A.2) by $T_{n}$ and integrating on $[-1,1]$ one gets, by the lower bounds (A.3) and (A.4), the uniform boundedness of $T_{n}$ and $u_{n}$ in the Sobolev norm of order 1 on $[-1,1]$, hence the compactness in $C([-1,1])$. Therefore the existence of solutions follows by choosing subsequences. The estimates (2.6) follow using the smoothness of $\eta$ and $\kappa$ and the lower bounds (A.3) and (A.4) in the explicit form of the solution:

$$
\begin{aligned}
u_{n}(y)= & -\mathscr{P} F \int_{-1}^{y} d y^{\prime} \eta_{n-1}^{-1}\left(y^{\prime}\right) \int_{-1}^{y^{\prime}} d y^{\prime \prime} T_{n-1}^{-1}\left(y^{\prime \prime}\right)+C_{1} \int_{-1}^{y} d y^{\prime} \eta_{n-1}^{-1}\left(y^{\prime}\right), \\
T_{n}(y)= & T_{0}-\int_{-1}^{y} d y^{\prime} \kappa_{n-1}^{-1}\left(y^{\prime}\right) \int_{-1}^{y^{\prime}} d y^{\prime \prime} \eta_{n-1}\left(y^{\prime \prime}\right)\left[\frac{d u_{n}}{d y}\left(y^{\prime \prime}\right)\right]^{2} \\
& +C_{2} \int_{-1}^{y} d y^{\prime} \kappa_{n-1}^{-1}\left(y^{\prime}\right)
\end{aligned}
$$

with $C_{1}$ and $C_{2}$ chosen so that the boundary conditions are satisfied also at $y=1$. $\eta_{n}$ and $\kappa_{n}$ denote here $\eta\left(T_{n}\right)$ and $\kappa\left(T_{n}\right)$. To show the uniqueness, let $\left(u^{(1)}, T^{(1)}\right)$ and $\left(u^{(2)}, T^{(2)}\right)$ be two solutions of (2.2), (2.3) with the given pressure $\mathscr{P}$, the boundary conditions (2.4), the lower bounds (A.3) and (A.4) and satisfying the estimates (2.6). Denoting by $w$ and $\sigma$ the differences $u^{(1)}-u^{(2)}$ and $T^{(1)}-T^{(2)}$ respectively, using above properties it is easy to prove that

$$
\int_{-1}^{1}\left\{\left[\frac{d w}{d y}\right]^{2}+\left[\frac{d \sigma}{d y}\right]^{2}\right\} d y \leqq c F \int_{-1}^{1}\left\{\left[\frac{d w}{d y}\right]^{2}+\left[\frac{d \sigma}{d y}\right]^{2}\right\} d y
$$


for a suitable constant $c$. This of course implies the uniqueness for small $F$. Finally we note that, to give a solution of the problem with fixed mass $m>0$, one has to solve the equation in $\mathscr{P}$

$$
\mathscr{P} \int_{-1}^{1} d y T_{\mathscr{P}}^{-1}=m
$$

where $T_{\mathscr{P}}$ denotes the solution with fixed pressure. Using Eqs. (A.5) and (A.6) it is not difficult to prove the solvability of this equation for $F$ small enough.

\section{Appendix B}

In this Appendix we prove the second part of Theorem 3.2. Moreover we check the conditions on the source terms in Eqs. (3.16) and (3.17) and on the boundary values that are needed in proving Proposition 3.1. First we state the following

Lemma B.1. The following identity holds for the Boltzmann collision operator (2.8):

$$
\frac{\partial}{\partial v} Q(f, g)=Q\left(f, \frac{\partial g}{\partial v}\right)+Q\left(g, \frac{\partial f}{\partial v}\right)
$$

where $\frac{\partial}{\partial v}$ stands for the partial derivative with respect to any of the components of $v$.

Proof. We observe that for any derivative, say $\partial_{v_{x}}$,

$$
\begin{aligned}
\frac{\partial}{\partial v_{x}} & \int_{\mathbb{R}^{3 \times S}} d v_{*} d \omega\left(v_{*}-v\right) \cdot \omega \chi\left(\left(v_{*}-v\right) \cdot \omega \geqq 0\right)\left[f^{\prime} g_{*}^{\prime}+g^{\prime} f_{*}^{\prime}-f g_{*}-g f_{*}\right] \\
\quad & =\int_{\mathbb{R}^{3 \times S}} d v_{*} d \omega \chi\left(\left(v_{*}-v\right) \cdot \omega \geqq 0\right)\left[\frac{\partial}{\partial v_{x}}+\frac{\partial}{\partial v_{* x}}\right]\left[f^{\prime} g_{*}^{\prime}+g^{\prime} f_{*}^{\prime}-f g_{*}-g f_{*}\right],
\end{aligned}
$$

$S$ being the unit sphere, since the function $\left(v_{*}-v\right) \cdot \omega \chi\left(\left(v_{*}-v\right) \cdot \omega \geqq 0\right)$ depends on the difference $v_{*}-v$. In (B.2) the notation is, as usual, $f, f_{*}, f^{\prime}, f_{*}^{\prime}$ for $f(v), f\left(v_{*}\right), f\left(v^{\prime}\right)$ and $f\left(v_{*}^{\prime}\right)$ respectively. Using the relations

$$
v^{\prime}=v+\omega\left[\omega \cdot\left(v_{*}-v\right)\right] ; v_{*}^{\prime}=v_{*}-\omega\left[\omega \cdot\left(v_{*}-v\right)\right]
$$

we get, for any function $h(v)$

$$
\begin{aligned}
& {\left[\frac{\partial}{\partial v_{x}}+\frac{\partial}{\partial v_{* x}}\right] h^{\prime}=\left(\frac{\partial h}{\partial v_{x}}\right)^{\prime},} \\
& {\left[\frac{\partial}{\partial v_{x}}+\frac{\partial}{\partial v_{* x}}\right] h_{*}^{\prime}=\left(\frac{\partial h}{\partial v_{x}}\right)_{*}^{\prime} .}
\end{aligned}
$$

The result then follows by a straightforward calculation. B.1,

In order to prove part 2) of Theorem 3.2 we differentiate (3.38) to get, by Lemma

$$
v_{y} \frac{\partial}{\partial \tau} \partial_{v} f=\mathscr{L}_{0} \partial_{v} f+2 Q\left(f, \partial_{v} M_{0}\right)+\partial_{v} s,
$$

where $\partial_{v} f$ stands for $\frac{\partial f}{\partial v_{x}}$. 
We claim that $\tilde{s}=2 Q\left(f, \partial_{v} M_{0}\right)+\partial_{v} s$ verifies the condition (3.44). In fact $\partial_{v} s$ satisfies (3.44) by the hypothesis (3.47). On the other hand

$$
\begin{aligned}
\sup _{\tau \in \mathbb{R}^{+}} & \sup _{v \in \mathbb{R}^{3}} e^{\sigma \tau}(1+|v|)^{r+1}\left|M_{0}^{-\frac{1}{2}} Q\left(f, \partial_{v} M_{0}\right)\right| \\
& \leqq \sup _{v \in \mathbb{R}^{3}}\left|(1+|v|)^{r} M_{0}^{-\frac{1}{2}} \partial_{v} M_{0}\right| \sup _{\tau \in \mathbb{R}^{+}} \sup _{v \in \mathbb{R}^{3}} e^{\sigma \tau}(1+|v|)^{r}\left|M_{0}^{-\frac{1}{2}} f\right| .
\end{aligned}
$$

The second factor in the r.h.s. of (B.7) is bounded by Theorem 3.2, part 1), while the first factor is obviously bounded. Besides, $\partial_{v} \vartheta(v)$ verifies (3.43) by the hypothesis (3.47). Therefore we can apply the first part of Theorem 3.2 to (B.6) and conclude that $\partial_{v} f$ exists and satisfies (3.48).

The proof of Theorem 3.2 is completed by the observation that the result for the derivative of any order is achieved following the same procedure, i.e. differentiating the equation for the derivative at the preceding order and controlling by direct inspection that the resulting equation has a source that satisfies condition (3.42) and (3.44) so that it is possible to apply part 1) of Theorem 3.2 to get the result. The same argument applies to $\frac{\partial f}{\partial v_{z}}$ but not to $\frac{\partial f}{\partial v_{y}}$, because in the latter case an extra term $\partial_{y} f$ would arise.

Now we verify that the source terms, that we denote by $s_{n}$, in (3.16) and (3.17) satisfy conditions (3.42), (3.44) and (3.48) in Theorem 3.2. The $s_{n}$ 's are defined by

$$
s_{2}=\left[2 Q\left(\Delta M, b_{1}^{-}\right)+2 Q\left(B_{1}, b_{1}^{-}\right)+Q\left(b_{1}^{-}, b_{1}^{-}\right)+Q\left(b_{1}^{-}, b_{1}^{+}\right)\right],
$$

and for $3 \leqq n \leqq 6$,

$$
\begin{aligned}
s_{n}= & -F \frac{\partial}{\partial v_{x}} b_{n-2}^{-}+2 Q\left(\Delta M, b_{n-1}^{-}\right)+\sum_{\substack{i, j \geqq 1 \\
i+j=n}}\left[2 Q\left(B_{i}, b_{j}^{-}\right)\right. \\
& \left.+Q\left(b_{i}^{-}, b_{j}^{-}\right)+Q\left(b_{i}^{+}, b_{j}^{-}\right)\right] .
\end{aligned}
$$

Property (3.42) is true for any $n$ due to the property (2.15) of $Q$. Since in $s_{2}$ all the terms are of the form $Q(f, g)$, they can be estimated using the Grad estimates (3.67). For the first term, for any $\sigma^{\prime}<\sigma$,

$$
\begin{aligned}
& \sup _{y^{\prime} \in \mathbb{R}^{+}} \sup _{v \in \mathbb{R}^{3}} e^{\sigma^{\prime} y^{\prime}}(1+|v|)^{r-1}\left|M_{0}^{-\frac{1}{2}} 2 Q\left(\Delta M, b_{1}^{-}\right)\right| \\
& \leqq c \sup _{y^{\prime} \in \mathbb{R}^{+}} \sup _{v \in \mathbb{R}^{3}}\left|M_{0}^{-\frac{1}{2}} \Delta M\right|(1+|v|)^{r} e^{-\left(\sigma-\sigma^{\prime}\right) y^{\prime}} \\
& \quad \times \sup _{y^{\prime} \in \mathbb{R}^{+}} \sup _{v \in \mathbb{R}^{3}} e^{\sigma y^{\prime}}\left|M_{0}^{-\frac{1}{2}} b_{1}^{-}\right|(1+|v|)^{r} \mid \\
& \leqq \frac{c F}{\left(\sigma-\sigma^{\prime}\right)},
\end{aligned}
$$

where the second inequality is obtained, as in (3.68), by means of the bound

$$
\sup _{y^{\prime} \in \mathbb{R}^{+}} \sup _{v \in \mathbb{R}^{3}} e^{\sigma y^{\prime}}(1+|v|)^{r}\left|M_{0}^{-\frac{1}{2}} b_{1}^{-}\right|<c
$$

that follows from Theorem 3.2 applied to Eq. (3.15). The other terms in $s_{2}$ are estimated in a similar and simpler way, by means of the bound on $B_{1}$ in Proposition 3.1 and the estimate (B.11). 
To verify property (3.48) for $s_{2}$, we apply Lemma B.1 to evaluate $\partial_{v} s_{2}$. Since all the terms in the expression of the derivative are of the form $Q\left(f, \partial_{v} g\right)$ we can bound $\sup _{y^{\prime} \in \mathbb{R}^{+}} \sup _{v \in \mathbb{R}^{3}} e^{\sigma^{\prime} y^{\prime}}(1+|v|)^{r-1}\left|M_{0}^{-\frac{1}{2}} \partial_{v} s_{2}\right|$ in terms of $\left|M_{0}^{-\frac{1}{2}} B_{1}\right|_{r}$,

$$
\sup _{y^{\prime} \in \mathbb{R}^{+}} \sup _{v \in \mathbb{R}^{3}}\left|M_{0}^{-\frac{1}{2}} \Delta M\right|(1+|v|)^{r} e^{-\left(\sigma-\sigma^{\prime}\right) y^{\prime}} \text { and } \sup _{y^{\prime} \in \mathbb{R}^{+}} \sup _{v \in \mathbb{R}^{3}} e^{\sigma y^{\prime}}\left|M_{0}^{-\frac{1}{2}} b_{1}^{-}\right|(1+|v|)^{r} \mid
$$

using again (3.67).

As far as $s_{n}$ is concerned we can estimate all the terms in the same way as for $s_{2}$, but the first one which involves the derivative of $b_{n-2}^{-}, n=3, \ldots, 6$. On the other hand we have already proven the estimate for the $v_{x}$-derivative of $b_{1}$ we need. Therefore we are in a position to proceed from now on iteratively to get the result.

Acknowledgements It is a pleasure to thank A De Masi, E. Presutti, M. Pulvirenti and L. Triolo for useful discussions. We thank the IHES in Bures-sur-Yvette and two of us (R.E. and R.M ) also the Math Department of the Rutgers University, where part of this work was done Research supported in part by AFOSR Grant 91-0010, MURST and GNFM.

\section{References}

1 Hilbert, D.: Grundzüge einer Allgemeinen Theorie der Linearen Intergral Gleichungen. New York: Chelsea 1953

2 Spohn, H.: Large Scale Dynamics of Interacting Particles Berlin, Heidelberg, New York: Springer 1991

3. Nishida, T.: Fluid dynamical limit of the nonlinear Boltzmann equation to the level of the compressible Euler equation. Commun. Math. Phys. 61, 119-148 (1978)

4. Caflisch, R E.: The fluid dynamic limit of the nonlinear Boltzmann equation. Commun. Pure Appl. Math. 33, 651-666 (1980).

5 Ukai, S.: The Euler limit and initial layer of the nonlinear Boltzmann equation Hokkaido Math. J. 12, 303-324 (1983).

6. Cercignani, C.: The Boltzmann Equation and Its Applications New York: Springer-Verlag 1988

7. De Masi, A, Esposito, R., Lebowitz, J.L : Incompressible Navier-Stokes and Euler limits of the Boltzmann equation Commun Pure Appl. Math 42, 1189-1214 (1989).

8. Bardos, C., Ukai, S.: The classical incompressible Navier-Stokes limit of the Boltzmann equation. Preprint, 1991

9. Asano, K.: The fluid Dynamical Limit of the Boltzmann Equation. Preprint, 1991

10. Bardos, C, Golse, F, Levermore, D.: Fluid dynamical limits of kinetic equations I Formal derivations. J. Stat. Phys. 63, 323-344 (1991)

11 Bardos, C, Golse, F., Levermore, D.: Fluid dynamical limits of kinetic equations II. Convergence proof for the Boltzmann equation Preprint, 1991

12. Esposito, R., Lebowitz, J L., Marra, R.: Navier-Stokes limit of the Boltzmann equation Preprint, 1991

13 Hannon, L., Lie, G C, Clementi, E: Micro-Hydrodynamics. J. Stat Phys 51, 965-979 (1988)

14. Bardos, C., Caflisch, R.E, Nicolaenko, B.: Thermal layer solutions of the Boltzmann Equation. In: Fritz, J., Jaffe, A. and Szasz, D. (eds.) Random Fields: Rigorous results in Statistical Physics. Proceedings, Koszeg 1984, Boston: Birkhauser 1985

15 Maslova, N B : Kramers problem in the kinetic theory of gases USSR Comput Math Phys. 22, 208-219 (1982)

16. Bardos, C., Caflisch, R.E., Nicolaenko, B.: The Milne and Kramers problems for the Boltzmann Equation of a hard sphere gas. Commun. Pure and Appl. Math XXXIX, 323-352 (1986)

17 Cercignani, C.: Half-space problems in the kinetic theory of gases. Lect. Notes in Phys. 249, Berlin, Heidelberg, New York: Springer 1986, pp 35-50.

18. Grad, A.: Asymptotic Theory of the Boltzmann equation. Phys. Fluids 6, 147-181 (1963) 
19. Grad, A.: Asymptotic Theory of the Boltzmann equation II. In: Rarefied Gas Dynamics II, Paris, 1962, pp. 26-59

20. Grad, A.: Asymptotic equivalence of the Navier-Stokes and nonlinear Boltzmann operator. Proc Symp. Appl. Math. XVII, 154-183 (1965)

21 Costantini, C., Marra, R.: Hydrodynamic Limits for the Boltzmann proc. J. Stat. Phys. 67, 229-249 (1992)

22 Golse, F., Perthame, B., Sulem, C.: On a boundary layer problem for the nonlinear Boltzmann equation. Arch. Rat. Mechanics 104, 81-96 (1988)

Communicated by Ya G. Sinai 